\title{
Experimental investigation of the stochastic nature of end-gas autoignition with detonation development in confined combustion chamber
}

Jianfu Zhao ${ }^{1}$, Lei Zhou ${ }^{1} *$, Lijia Zhong ${ }^{1}$, Xiaojun Zhang ${ }^{1}$, Jiaying Pan $^{1}$, Rui Chen $^{1,2}$, Haiqiao Wei ${ }^{1, *}$ 1, State Key Laboratory of Engines, Tianjin University, Tianjin 300072, China

2, Department of Aeronautical and Automotive Engineering, Loughborough University, Loughborough LE11 3TU, United Kingdom

*Corresponding author:

Address: 92 Weijin Road, Nankai District, Tianjin, P. R. China

Tel.: +86-22-27402609

Email: lei.zhou@tju.edu.cn(L. Zhou)

whq@tju.edu.cn(H.Wei)

\begin{abstract}
In the present work, end-gas autoignition formation, and the effects of oxygen concentration on the flame/shock waves propagation and pressure oscillation, are investigated in a self-designed constant-volume chamber equipped with a perforated plate. A hydrogen-oxygen-nitrogen mixture with adjustable oxygen to nitrogen ratio is chosen as the test fuel. In an oxygen-enriched condition, the probability of an end-gas autoignition occurrence increases significantly. End-gas autoignition with detonation development is further investigated, with a special emphasis on the stochasticity of the detonation development. In a low-oxygen condition, detonation occurs randomly owing to its stochastic physical behavior. However, when the oxygen concentration increases to $28 \%$, the stochastic factors have a lower impact, and the detonation occurrence is certain. Nevertheless, the pressure and pressure oscillation in the autoignition exhibit random behaviors and are unrelated to the oxygen concentration. The variation tendency of the flame tip velocity remains constant under different oxygen concentrations. However, an increase in the oxygen concentration improves the flame tip velocity, thereby inducing stronger shock waves and promoting autoignition. Based on the start time of the autoignition, two types of autoignition modes were identified: Mode 1 and Mode 2. In Mode 2, the unburnt mixture experiences merely one compression by the shock wave before autoignition and only occurs under high oxygen concentrations of $27 \%-28 \%$. Under equal oxygen concentrations, the pressure and pressure oscillation of Mode 2 are higher than those of Mode 1 owing to the larger amount
\end{abstract}


of unburnt mixture. Finally, the exhaust gas was introduced into the initial unburnt mixture to investigate the effect of an inert gas on the combustion.

Key words: End-gas autoignition; Detonation; Oxygen concentration; Pressure oscillation; Confined space

\section{Introduction}

The understanding of premixed-combustion dynamics in a confined space is essential for a wide range of combustion processes and applications, including gas explosions in confined regions and the energy production in internal combustion engines [1-4]. Depending on the reactive material and confinement geometry [5-8], the initial laminar flame can evolve into a turbulent flame, or even experience a deflagration to detonation transition (DDT), which remains one of the most intriguing and least understood effects in hydrodynamics, combustion science, nonlinear physics, and astrophysics [9]. A controlled detonation initiation in certain propulsion systems could revolutionize transportation (e.g. pulse detonation engines (PDEs)), whereas uncontrolled detonations can destroy facilities and result in disasters [10-13], such as gas explosions during mining operations and knocks in downsized spark ignition engines.

A qualitative explanation of the DDT process was first provided by Shelkin [14]. A detonation occurs if the reaction is sufficiently energetic to form a shock wave ahead of the flame and the shock is sufficiently strong to auto-ignite the gas mixture before it is approached by the following flame. In [15], it is demonstrated that a flame can accelerate more rapidly if it passes through an array of turbulence generators, including orifices, baffles, and spirals. Lee et al. [16] reported that a quasistationary flame speed can be attained after a distance of 30-40 duct diameters. A high flame speed creates a high gas velocity ahead of the flame, thereby improving the strength of the shock wave and, most importantly, the pressure and temperature behind the shock wave. Therefore, the autoignition delay time might become sufficiently small to induce autoignition and DDT. However, the previously mentioned investigations were carried out in semi-open or vented obstructed channels (where one end of the channel is closed and the flame is ignited at the closed end and moves toward the open end). In a confined space, such as internal combustion engines, the combustion physics induced by flame-shock wave or compression wave interactions become increasingly complicated.

In a downsized spark ignition engine, knocking combustion is very likely, which constrains the thermal efficiency and even causes irreversible damages to the engine [17-20]. Particularly, in the lowand high-load operating regimes, a super-knock can result in very strong pressure oscillations with a high peak pressure ( $>300$ bar). The major source of knocking combustion is a pressure wave caused by end-gas autoignition; i.e., autoignition of the unburnt mixture present between a propagation flame 
and the engine wall. By focusing on an end-gas autoignition event and the subsequent pressure wave development, extensive one-dimensional numerical investigations were conducted to study the behaviors of the reaction front propagation from hot (or cold) spots. Zeldovich [21] reported different combustion modes for a flame front propagating in a charge with non-uniform reactivity. Bradley, $\mathrm{Gu}$, and Kalghatgi [22-24] developed an operational peninsula based on the dimensionless parameters $\xi$ and $\varepsilon$, which represent the normalized temperature gradient and the ratio of acoustic time to excitation time, respectively, to describe the limits of a developing detonation mode based on $\mathrm{H}_{2}-\mathrm{CO}-$ air and $\mathrm{H}_{2}$-air mixtures. Dai et al. [25] identified different autoignition modes caused by a cool spot with a positive temperature gradient, thereby suggesting its essential role for the autoignition heat release and pressure wave in the subsequent evolution of the combustion behavior. However, these studies require the artificial introduction of a temperature gradient and lack a flame propagation model.

To overcome this, Robert et al. [26] used 15 large eddy simulation (LES) cycles for highload/low-speed spark ignited engine operations to visualize knocking and super-knocking combustions. Further, they indicated that the coupling between the pressure wave and autoignition reaction reinforces each other, thereby leading to maximum pressures and propagation speeds close to the detonation. Terashima et al. [27] simulated a complete knocking combustion process from the flame propagation to the pressure wave generation associated with the end-gas autoignition and clarified the mechanisms of the hot-spot formation and pressure wave development. In their experimental studies, Kawahara et al. [28] observed end-gas autoignition during knocking combustion in a compressionexpansion engine. By comparing the pressure oscillations, thermodynamic states, and pressure rises relative to an isochoric combustion with those of a detonation in a rapid compression machine (RCM), Qi et al. [29] confirmed that a super-knock is the result of a detonation. Inside the engine and RCM, the flame front and flow are three-dimensional and thereby it is difficult to understand the interaction between pressure wave and chemical reaction during the autoignition process.

Despite the numerous studies devoted to investigating DDT and knocking combustion, fundamental problems remain regarding the formation of the end-gas autoignition and the interaction between the flame and pressure waves. Because it is a small-scale transient phenomenon, end-gas autoignition during knocking combustion is difficult to measure even with various experimental techniques. In our previous studies [30, 31], the entire evolution of an end-gas autoignition with detonation development was observed, which was caused by flame-shock interactions in a constantvolume chamber with a perforated plate. Nevertheless, the end-gas autoignition appears to be intermittent due to the stochastic nature of DDT. Similarly, the run-up distance to the DDT in channels with obstacles can vary considerably from one experiment to another; even though great efforts were devoted to ensuring equal experimental conditions [32]. Therefore, the fundamental mechanism has 
not been carefully investigated yet. It is worth stressing that the occurrence of knocking combustions in practical spark ignition engines is a stochastic process. This is normal for a complex physical system that involves multiple stochastic phenomena, including flow instabilities; turbulence; many interactions between shocks, flames, and vortices; and the resulting hot-spot formation.

The objective of this study is to investigate the mechanism of end-gas autoignition formation with a detonation development considering the stochasticity of DDT. To the best of the authors' knowledge, this is the first comprehensive analysis of the effects of oxygen concentration on the flame propagation, shock wave propagation, and end-gas autoignition in a confined space, which is useful to understand the stochasticity and inevitability of the end-gas autoignition. The stochastic nature of turbulent flames and end-gas autoignitions with detonation waves has not been considered in previous studies. In this study, a hydrogen-oxygen-nitrogen mixture was used with adjustable oxygen to nitrogen ratio. In addition, the exhaust gas with the remaining water component of the combustion production was introduced into the reaction, and the effect of the exhaust gas ratio was discussed. This study provides new insight into not only the knocking combustion and DDT phenomenon in engines and industries, but also into the fundamentals of turbulent combustion.

The rest of this paper is organized as follows. The effects of the oxygen concentration on the flame and shock wave propagations, pressure oscillation, and end-gas autoignition are discussed first. Then, the formation of end-gas autoignition with different detonation development modes is explained, followed by a comparison of normal combustion and end-gas autoignition in terms of pressure and flame tip velocity. Finally, the influence of the introduced exhaust gas on the combustion phenomenon is analyzed. 


\section{Experimental apparatus and procedure}

\subsection{Experimental apparatus}

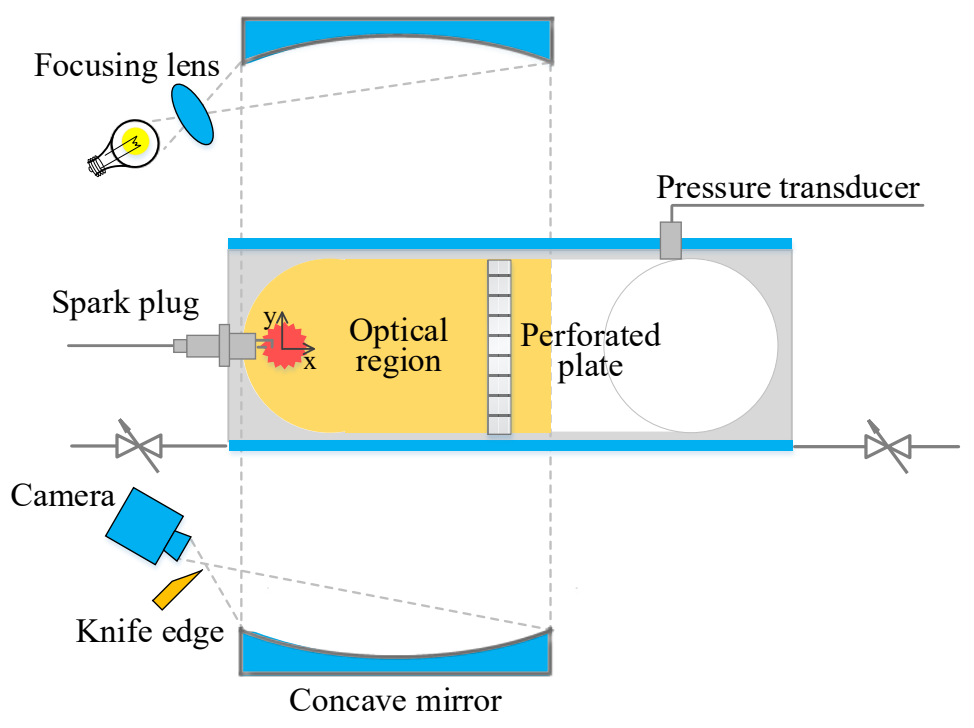

(a) Scheme A: experimental setup for capturing images on the left side of the perforated

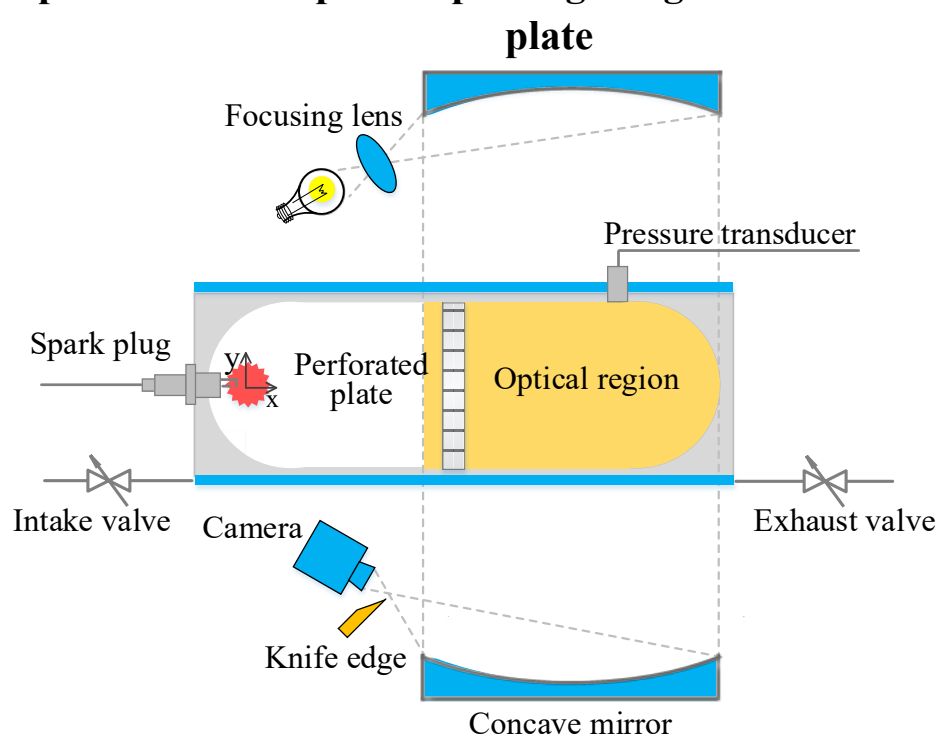

(b) Scheme B: experimental setup for capturing images on the right side of the perforated

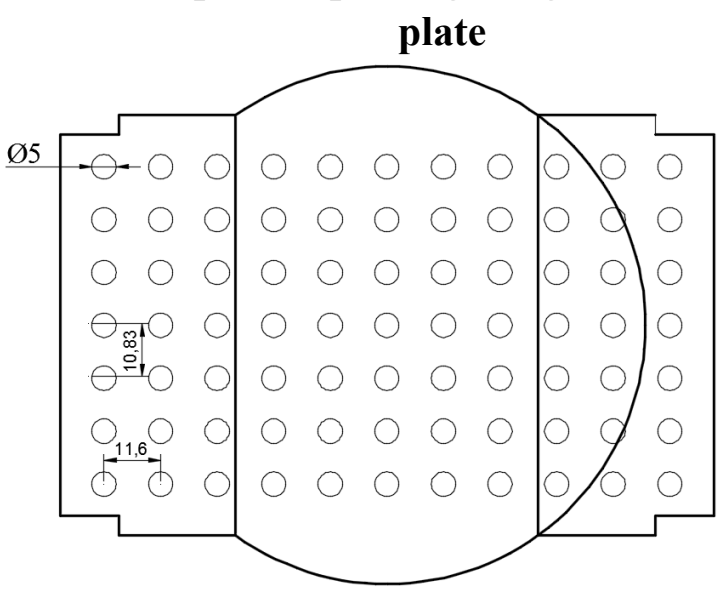




\section{(c) Perforated plate}

\section{Fig. 1 Schematic of the experimental setup}

In the present study, a self-developed constant-volume combustion bomb (CVCB) (Fig. 1) was employed. The experimental setup is composed of a combustion chamber, perforated plate, intake and exhaust system, ignition system, heating system, time synchronization system, and image acquisition system. The combustion chamber is composed of a cylinder (100 mm diameter and $230 \mathrm{~mm}$ length) with a total volume of $2.32 \mathrm{~L}$. Two optical glass windows are mounted, one in the front and one in the back of the CVCB. Owing to the light penetration through the optical windows and combustion chamber, the chamber is not a complete cylinder. The windows are made of high-quality quartz glass, which can provide optical access. The windows are in a racetrack shape $230 \mathrm{~mm}$ in length and $80 \mathrm{~mm}$ in width. As illustrated in Fig. 1 (c), the perforated plate is a 3-mm-thick steel plate with several holes fixed at the combustion chamber center to accelerate the flame and promote a turbulent flame and shock wave formation. The hole size and porosity of the perforated plate are $5 \mathrm{~mm}$ and $12 \%$, respectively. The lateral distance of the two adjacent columns cores is $11.6 \mathrm{~mm}$, while the longitudinal distance of two adjacent rows cores is $10.83 \mathrm{~mm}$. A cluster of electric heating units with a total power of $2 \mathrm{~kW}$ was used to heat the entire CVCB evenly. The bomb temperature was controlled within $3 \mathrm{~K}$ with a closed-loop feedback controller, which prevented combustion products from condensing into droplets. A Bosch R6 spark plug, which can generate a spark with a duration of $0.7 \mathrm{~ms}$ and energy of $30 \mathrm{~mJ}$, was employed to ignite the mixture. The pressure was recorded using a pressure sensor (Kistler $6045 \mathrm{~A}$ ) with a natural frequency of $80 \mathrm{kHz}$. After passing through a charge amplifier (Kistler 5018), the pressure signals were recorded with a National Instruments USB-6361 data acquisition system (16 bit, $2 \mathrm{M}$ samples/s) with a frequency much higher than that of the transducer to avoid overlapping. The combustion images were recorded via high-speed Schlieren photography at a frame rate of 70,000 fps and exposure time of $1 \mu \mathrm{s}$. A time synchronization system was applied to guarantee that the image acquisition system, pressure transducer, and ignition spark operated synchronously.

\subsection{Experimental procedures}

As illustrated in Fig. 1, the maximum optical region for the high-speed Schlieren photographs is a circle with a diameter of $150 \mathrm{~mm}$, which is smaller than the combustion chamber. Therefore, the two arrangements illustrated in Fig. 1 (a) and (b) were applied to obtain the flame propagation in front of and behind the perforated plate, respectively. Further, a hydrogen-oxygen-nitrogen mixture was used; the ratio of the oxygen to nitrogen was adjustable. In the present study, the oxygen concentration was defined as the ratio of $\mathrm{O}_{2}$ mol to $\left(\mathrm{O}_{2}+\mathrm{N}_{2}\right)$ mol:

$$
\text { Oxygen concentration }=\frac{m_{O_{2}}}{m_{O_{2}}+m_{N_{2}}} \text {. }
$$


The hydrogen, oxygen, and nitrogen quantity is determined based on the Dalton's law of partial pressure. The measurement accuracy of the pressure transmitter is $0.25 \%$, which ensures the precise control of the oxygen concentration. Note that the $\mathrm{H}_{2}-\mathrm{O}_{2}-\mathrm{N}_{2}$ mixture is stoichiometric under an oxygen concentration of $19 \%$. As the oxygen concentration increases, the amount of hydrogen (i.e. energy density) and total pressure remain constant. As a result, the mixture becomes lean with increasing oxygen concentration. The equivalence ratios under oxygen concentration of $19 \%, 21 \%$, $23 \%, 25 \%, 26 \%, 27 \%$, and $28 \%$ were $1,0.911,0.831,0.765,0.736,0.708$, and 0.683 , respectively. The initial experimental conditions are listed in Table 1. Note that high oxygen concentrations and even pure fuel-oxygen systems are generally used to quickly obtain a DDT process in most studies [33-35]. In this study, a high oxygen concentration was used to analyze the in-depth mechanisms of end-gas autoignition in a confined space.

Table 1 Test conditions

\begin{tabular}{ll}
\hline Item & Value \\
\hline Hole size $/ \mathrm{mm}$ & 5 \\
Porosity $/ \%$ & 12 \\
Initial temperature $/ \mathrm{K}$ & $363 \pm 3$ \\
Initial pressure $/$ bar & 3 \\
Oxygen concentration $/ \%$ & $19,21,23,25,26,27,28$ \\
\hline
\end{tabular}

\subsection{Definition of velocity}

In this study, the velocity of the flame was defined as the flame tip velocity (absolute velocity) in the laboratory frame to indicate how rapidly the leading edge would traverse a certain distance. The velocity is calculated based on the time derivation of the flame tip position, which is the distance from the ignition point (Eq. (2)). It should be noted that this flame tip velocity is not the actual burning velocity but a superposition of the burning velocity and flow velocity. However, this velocity is used extensively in investigations on flame acceleration and DDTs [2, 15, 30, 36-39].

$$
\text { Flame tip velocity }=\frac{D_{i+1}-D_{i}}{T_{i+1}-T_{i}}
$$

Where $D_{i}$ represents the distance from the tip to the ignition point at time $T_{i}$.

In the current data, the spatial resolution of the flame tip velocity measurement is $0.18 \mathrm{~mm}$. Consequently, based on a frame rate of $70,000 \mathrm{fps}$, a misinterpretation of one pixel represents an uncertainty of $12.6 \mathrm{~m} / \mathrm{s}$ in the velocity. 


\section{Results and discussions}

\subsection{Stochastic characteristics}

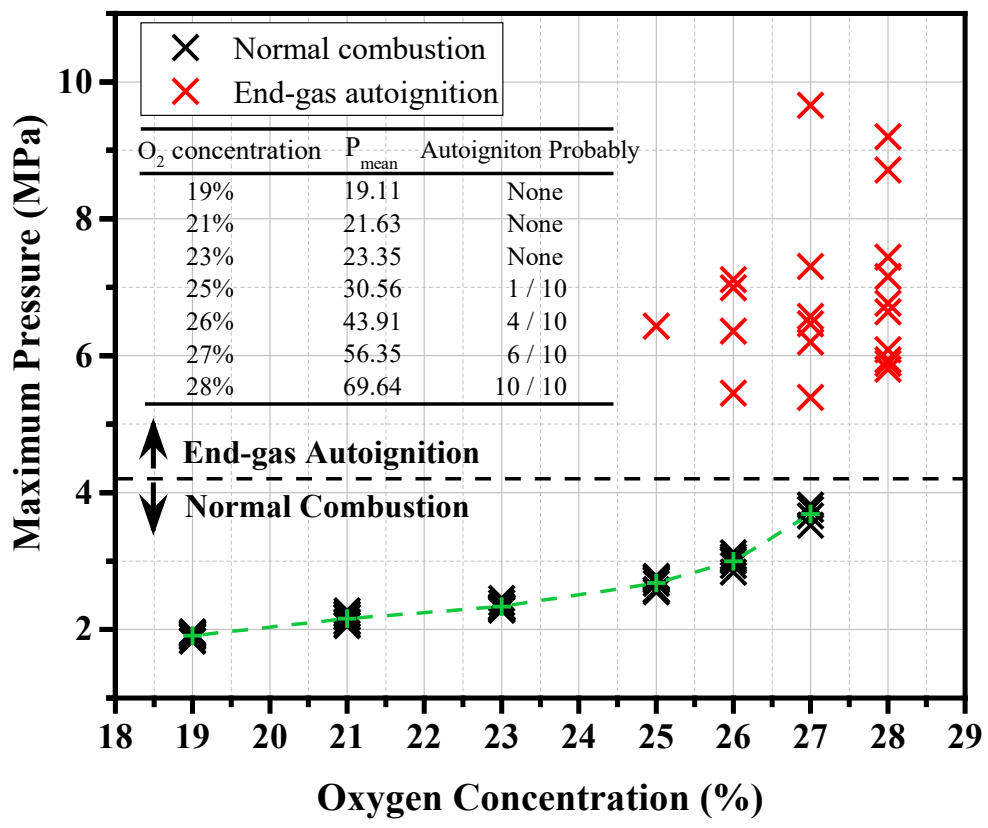

Fig. 2 Evolution of maximum pressure and combustion mode under different oxygen concentrations ( $P_{\text {mean }}$ represents the mean value of the maximum pressure of all ten cases, while the green cross indicates the mean value of the maximum pressure of normal combustion cases under a specific oxygen concentration)

In this study, ten repeated tests were carried out under each oxygen concentrations, which was changed between $19 \%$ and $28 \%$. The initial 3-bar pressure of the combustible mixture and hydrogen amount remained constant in all experiments. The experimental results, including the maximum pressure, mean pressure, and combustion modes, are summarized and illustrated in Fig. 2. The mean value of the maximum pressure of the normal combustion cases under a specific oxygen concentration is marked by green cross in Fig. 2. It is observed that maximum pressure of normal combustion under a specific oxygen concentration remains the same, which verifies the repeatability of the experiments. The combustion modes were distinguished based on the combustion Schlieren images and classified as normal combustion without end-gas autoignition (represented by black crosses) and end-gas autoignition (represented by red crosses). Under oxygen concentrations of $19 \%, 21 \%, 23 \%, 25 \%, 26 \%$, and $27 \%$, the mean value of the maximum pressure of the normal combustion (represented by green crosses) is 1.91, 2.16, 2.33, 2.68, 3.00, and 3.69 MPa, respectively. As shown in Fig. 2, the mean value of the maximum pressure of the normal combustion and the probability of end-gas autoignition occurrence increase with increasing oxygen concentration. A detailed explanation is provided in the 
following section.

As illustrated in Fig. 2, with oxygen concentrations of $19 \%, 21 \%$, and $23 \%$, only normal combustion mode exists. With an oxygen concentration of $25 \%$, the combustion mode with end-gas autoignition starts to occur. Note that the end-gas autoignition will induce a detonation development, thereby resulting in an extremely high peak pressure compared with that of the normal combustion mode (discussed in a later section). The end-gas autoignition with detonation development appears stochastically under these conditions. The stochastic occurrence is a natural behavior for a complex physical system that involves multiple stochastic phenomena, including flow instabilities; turbulence; many interactions between shocks, flames, and vortices; and a resulting hot-spot formation. Gamezo et al. [32] pointed out that small fluctuations in the thermal conditions have a significant impact on the stochastic nature of DDT. Further, they conducted simulations in which they exchanged the initial ambient temperature with an interval of $0.01 \mathrm{~K}$ to estimate the stochastic DDT behavior.

Although the stochastic nature of end-gas autoignition can be observed, as the oxygen concentration increases from $25 \%$ to $28 \%$, the probability of the end-gas autoignition occurrence increases (Fig. 2). The probability increases from 1 in 10 at $25 \%$ to 10 in 10 at $28 \%$. There are two possible reasons for this phenomenon. Firstly, with increasing oxygen and decreasing nitrogen concentrations, molecular collisions between hydrogen and oxygen are promoted, which enhance the potential reaction rate. Secondly, the higher burning temperature with increasing oxygen concentration introduces a stronger heating effect on the unburnt mixture, and provides more appropriate initial reaction conditions. Although the probability of the end-gas autoignition occurrence increases with increasing oxygen concentration, the maximum pressure of the end-gas autoignition mode exhibits random characteristics and is unrelated to the oxygen concentration. Furthermore, an interesting combustion phenomenon is observed: the occurrence of an end-gas autoignition with detonation changes from a stochastic process to an unavoidable event, which differs from the stochastic process of a DDT presented by Gamezo et al. [32]. Therefore, the thermodynamic state may finally determine whether an end-gas autoignition occurs under certain conditions. Overall, the competition between the stochastic nature of the turbulent combustion and detonation occurrence and the effect of the high reactivity of the thermodynamic condition might be responsible for the end-gas autoignition in a confined space. 


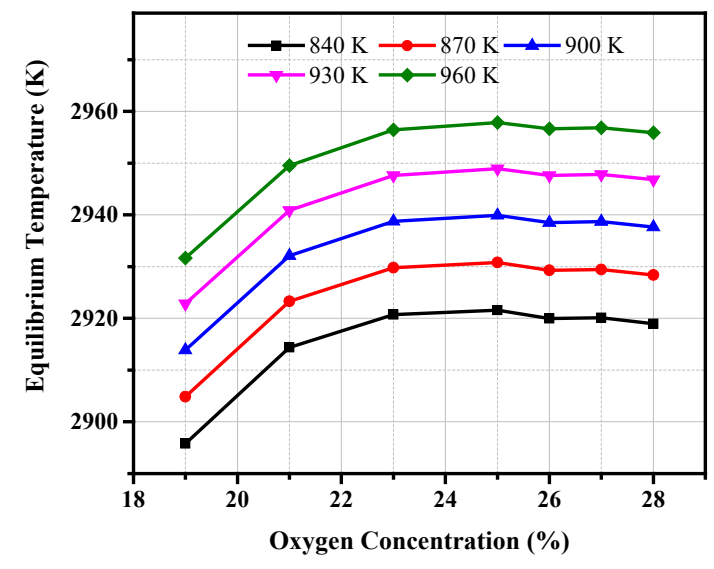

(a) Equilibrium temperature under different oxygen concentrations

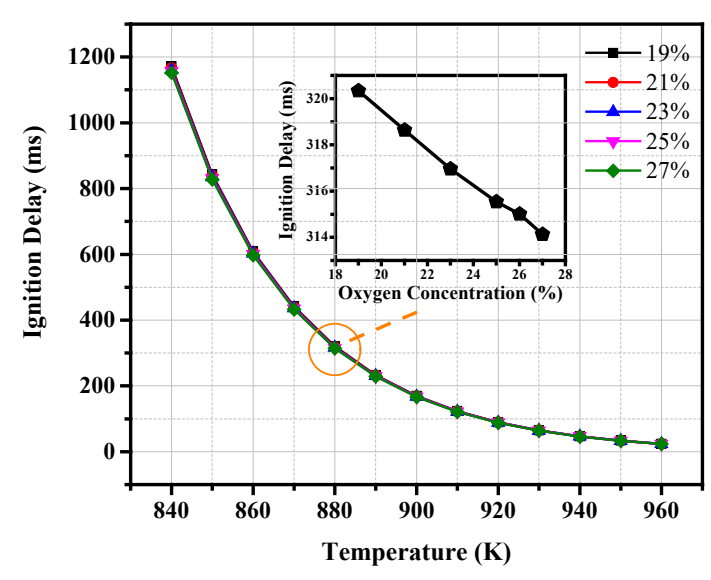

(b) Ignition delay time under different oxygen concentrations

\section{Fig. 3 Calculated equilibrium temperature and ignition delay via zero-dimensional simulation}

To analyze the effect of the oxygen concentration on the combustion process, a zero-dimensional simulation (based on the Chemkin software) was performed by using a detailed reaction mechanism for $\mathrm{H}_{2}$ /air mixture. The resulting equilibrium temperature and ignition delay time of the $\mathrm{H}_{2}-\mathrm{O}_{2}-\mathrm{N}_{2}$ mixture are illustrated in Fig. 3. The initial pressure is 3 bar. According to Fig. 3 (a), the equilibrium temperature firstly increases sharply with increasing oxygen concentration and then remains approximately constant. The combustion temperature firstly increases with increasing oxygen concentration, which leads to a high radiative heat transfer to the unburnt mixture. However, excessive oxygen concentration enhances the dissociation reaction, which has an inhibiting effect on the burning temperature. As shown in the Fig. 3 (b), the curves under different oxygen concentrations appear to overlap, which means that the oxygen concentration (at constant temperature) has only a minor influence on the ignition delay. A sub-picture is added, from which it is observed that the ignition delay decreases with the increase of oxygen concentration. To summarize, the changes in the oxygen concentration cause the changes in equilibrium temperature and ignition delay, which further influence the flame propagation and end-gas autoignition. And it seems that the change in equilibrium temperature is the dominant factor contributing to or mitigating DDT. Based on the numerical results, the influences of oxygen concentration on the flame/shock propagation and pressure oscillation are discussed in the following sections. 


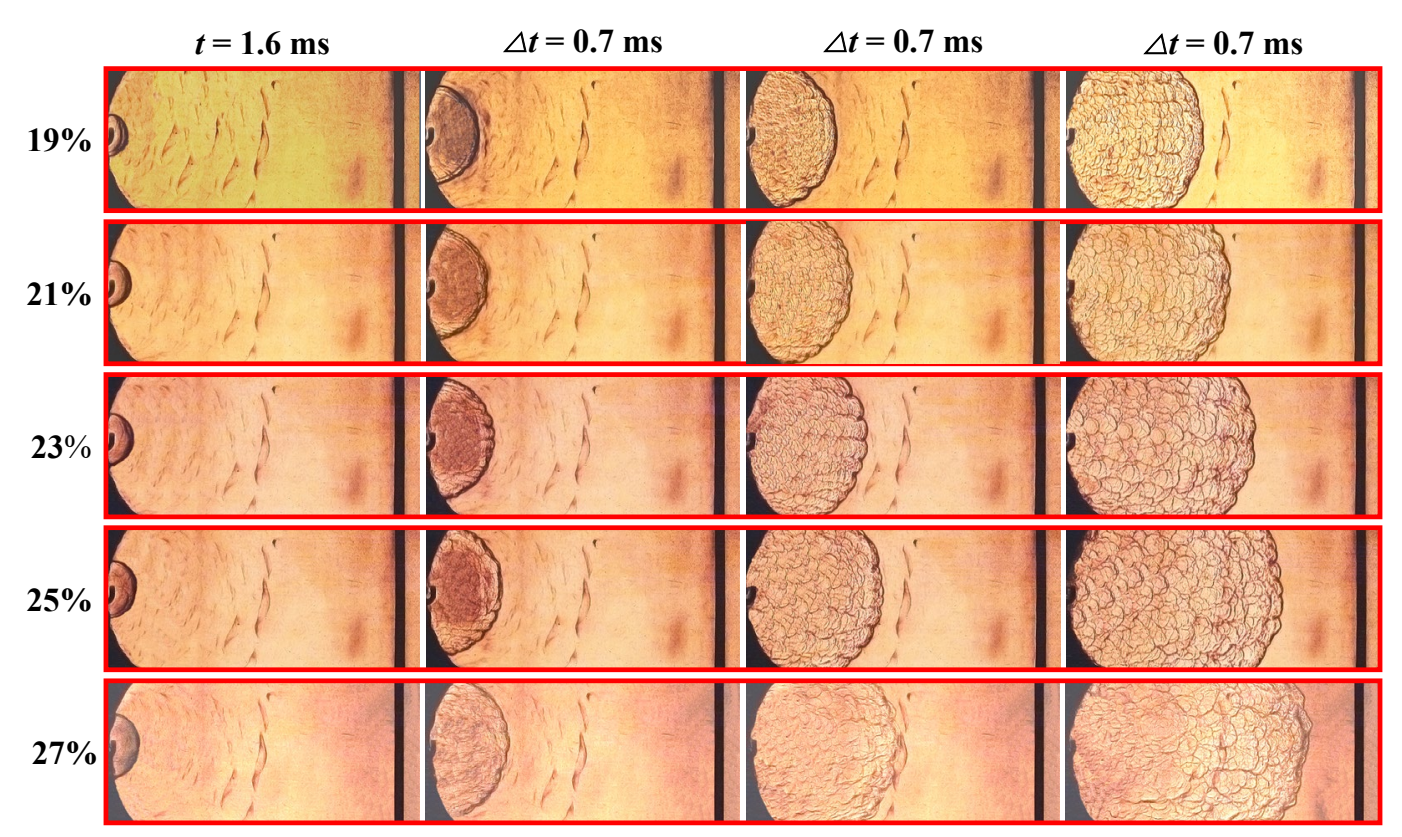

\section{Fig. 4 Sequential Schlieren images of flame development before the perforated plate under different oxygen concentrations}

A sequence of high-speed Schlieren images of the flame development before the perforated plate under oxygen concentrations of $21 \%-27 \%$ is illustrated in Fig. 4 . The noise-like dark fringes on the left side of the images are the tiny cracks in the inner surface of the quartz window (caused by the intense impacts of the detonation waves). As illustrated in Fig. 4, the flame is ignited on the left side of the image and propagates toward the right. When the mixture was ignited, the flow field was considered to be steady and without turbulences in our experiment. After the spark ignition, a smooth spherical flame is induced, which spreads outward. As time passes, a cellular structure appears on the flame surface caused by the Darrieus-Landau instability and thermal-diffusion instability [1, 15]. A video is provided as Supplemental Material (called "Flame Propagation before the Perforated Plate"), which shows the flame evolution in detail. Based on the video, it was found that the cellular structure appears earlier under higher oxygen concentrations. As previously described, the initial pressure of the combustible mixture and the amount of hydrogen remain the same at different oxygen concentrations. The flammable mixture is stoichiometric only under an oxygen concentration of $19 \%$. As the oxygen concentration increases, the equivalence ratio of the unburnt mixture decreases to a lean condition. According to Sun et al. [40], as the oxygen concentration increases, the effective Lewis number decreases to values below 1, which implies a stronger thermal-diffusion instability, and consequently an earlier occurrence of a cellular structure on the flame surface.

The first selected Schlieren image for every oxygen concentration in Fig. 4 is at time $t_{1}=1.6 \mathrm{~ms}$ 
after the spark ignition. The time interval between consecutive Schlieren images was kept constant $(\Delta t=0.7 \mathrm{~ms})$. The displacement of the flame front from the spark plug increases with increasing oxygen concentration. Thus, the flame propagation velocity is positively related to the oxygen concentration. This is because the increased oxygen concentration promotes molecular collisions and consequently increases the burning rate.

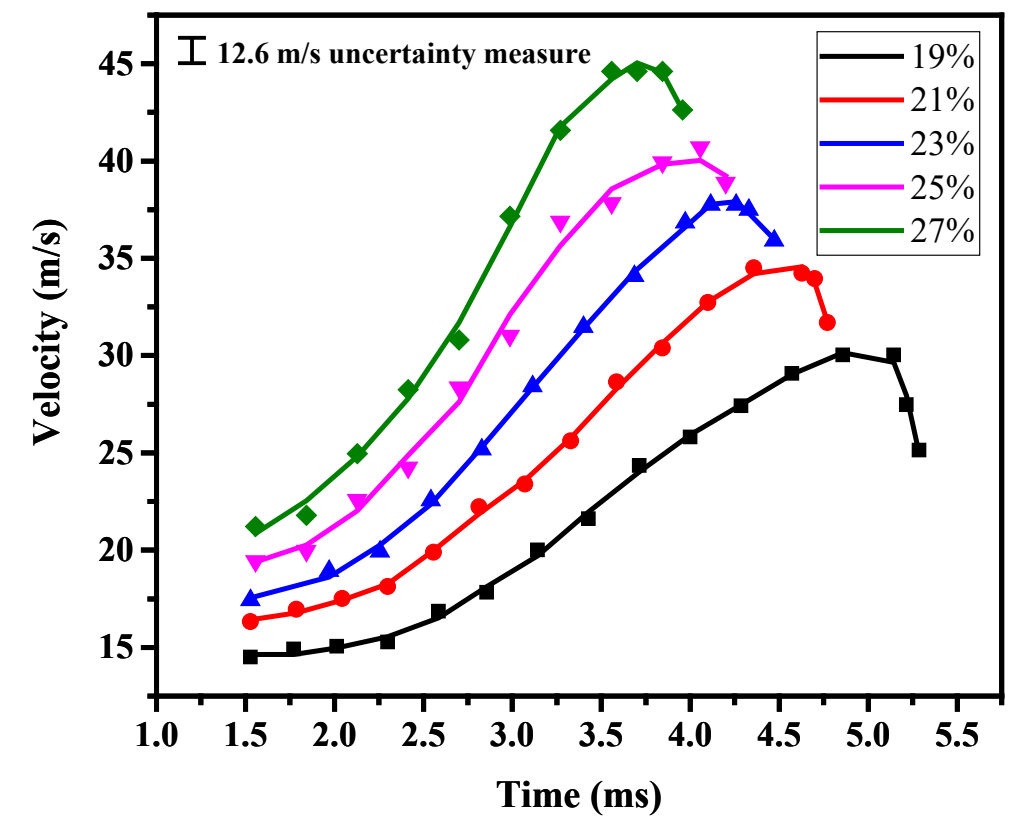

Fig. 5 Flame tip velocity versus time before the flame passes through the perforated plate under different oxygen concentrations

In our previous work [30], a repeatability test conducted based on the flame propagation demonstrated that the flame propagation remains unchanged in terms of tendency and velocity under the same initial conditions. Thus, in the present work, one case under a specific oxygen concentration is chosen for comparison, which is representative of the ten repeated experiments. Figure 5 illustrates the evolution of the flame tip velocity before the perforated plate with time under different oxygen concentrations. The velocity was calculated based on the time derivation of the flame tip position (the distance from the ignition point). A velocity point is calculated from the difference in the centerline flame position between two consecutive frames. In the current data, the spatial resolution of the flame tip position measurements is $0.18 \mathrm{~mm}$. Consequently, based on a framing rate of $70,000 \mathrm{fps}$, a misinterpretation of one pixel represents an uncertainty of $12.6 \mathrm{~m} / \mathrm{s}$ in the velocity. As illustrated in Fig. 5, the variation tendency of the flame tip velocity remains constant under different oxygen concentrations: the flame tip velocity initially increases and then decreases. The initial acceleration can be related to the effect of the Darrieus-Landau instability and thermal-diffusion instability, which wrinkle the flame surface to a cellular structure and cause an increase in the flame surface area. The 
energy generation and flow ahead of the flame accelerate when the flame surface area increases. Then, as the flame approaches the perforated plate, the flame decelerates owing to the influence of gas compression due to the confinement. The compression waves induced by the flame will be reflected from the perforated plate and reverse the flow of gas in front of the propagating flame, which consequently causes the flame tip velocity to decrease. It is noted that the actual burning velocity does not slow down. More detailed explanations about the flame propagation can be found in [30]. As concluded from the Schlieren images of Fig. 4, Fig. 5 evidently implies that the flame tip velocity increases with increasing oxygen concentration. In the present experiments, with a limited oxygen concentration range, the flame tip velocity before the flame passes through the perforated plate is positively related to the oxygen concentration. Bases on the numerical results shown in Fig. 3, the increasing oxygen concentration causes the increase in the equilibrium temperature and decrease in ignition delay, which consequently enhances the reactivity of the mixture and promotes the flame propagation.
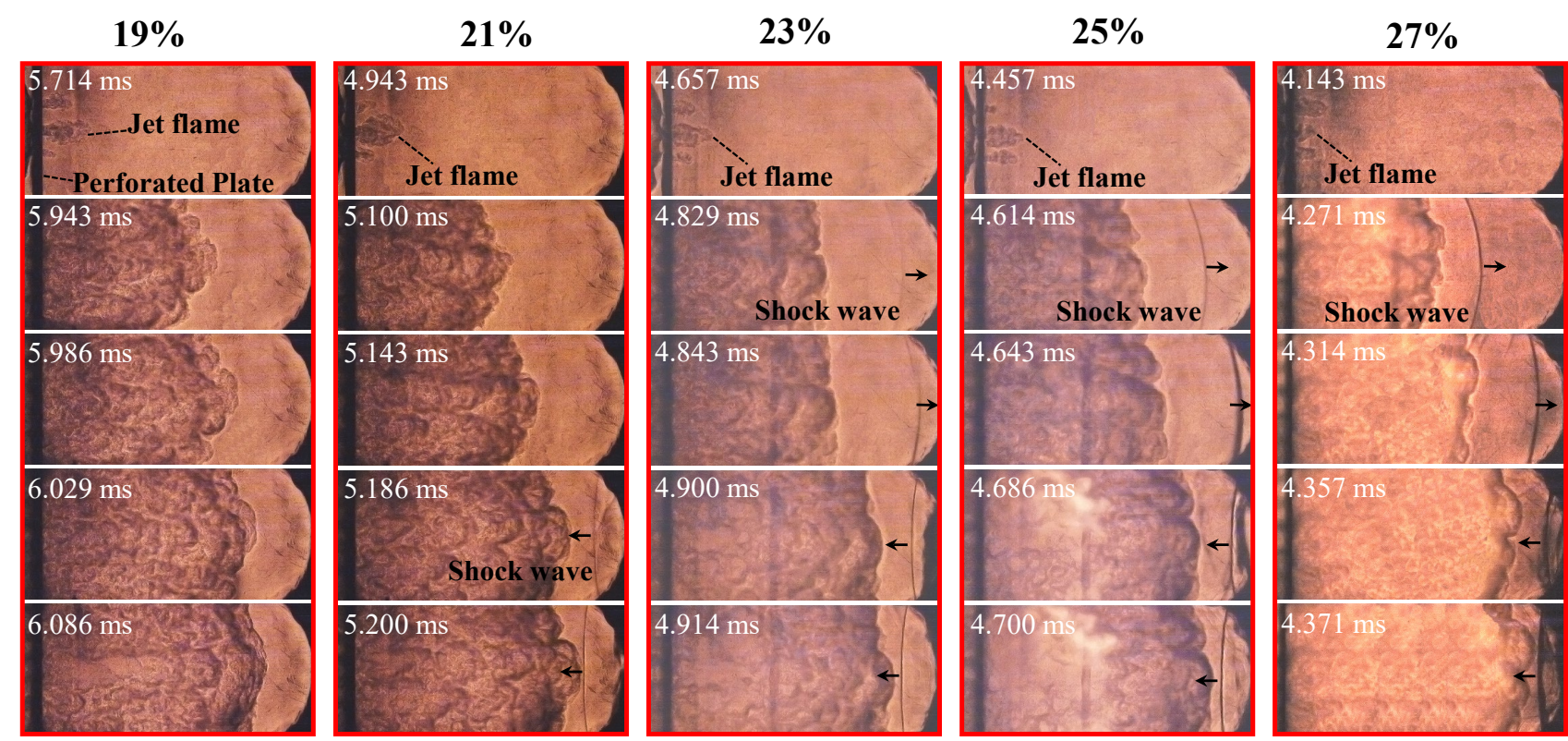

Fig. 6 Sequential Schlieren images of turbulent flame propagation behind the perforated plate under different oxygen concentrations

Fig. 6 illustrates the chronological sequence of the Schlieren images of the effects of the oxygen concentration on the turbulent flame propagation after the flame passes through the perforated plate. Figure 7 illustrates the variations in the flame tip velocity versus time under different oxygen concentrations after the flame passes through the perforated plate. It is noted that flame propagation remains consistent among ten repeated experiments under a specific oxygen concentration. Thus, the chosen case is representative of all ten experiments under a specific oxygen concentration. As 
illustrated in Fig. 6, after passing through the perforated plate, the flame is split into several jet flames. Then, the jet flames coalesce with each other, and a wrinkled turbulent flame is formed. Compressed by the accelerated flame, a shock wave is formed in the unburnt mixture. In our previous study [47], the formation and propagation evolution of the shock wave were discussed in detail. In this study, the effects of the oxygen concentration on the combustion phenomena involving the accelerated flame propagation and shock wave are the main focus. As illustrated in Fig. 6, no visible shock wave is observed under the oxygen concentration of $19 \%$. However, ahead of the flame front, there may exist compression waves that are not strong enough to form a visible shock wave. When the oxygen concentration increases to $21 \%$, a left-traveling shock wave, which is reflected at the end wall, is observed. Under oxygen concentrations of $23 \%, 25 \%$, and $27 \%$, right- and left-traveling shock waves are observed in the unburnt mixture. Further, when the oxygen concentration increases from $23 \%$ to $27 \%$, the color depth of the shock wave becomes increasingly darker, which implies a stronger intensity of the shock wave [30]. The shock wave intensity increases with increasing oxygen concentration, which results in a strong compression of the unburnt mixture, and consequently increases the local pressure and temperature. Therefore, it is evident why the increased oxygen concentration promotes the end-gas autoignition. However, the process deserves further investigation with advanced measurement methods to obtain the transient pressure and temperature of the unburnt temperature. These might offer a thorough understanding of the end-gas autoignition.

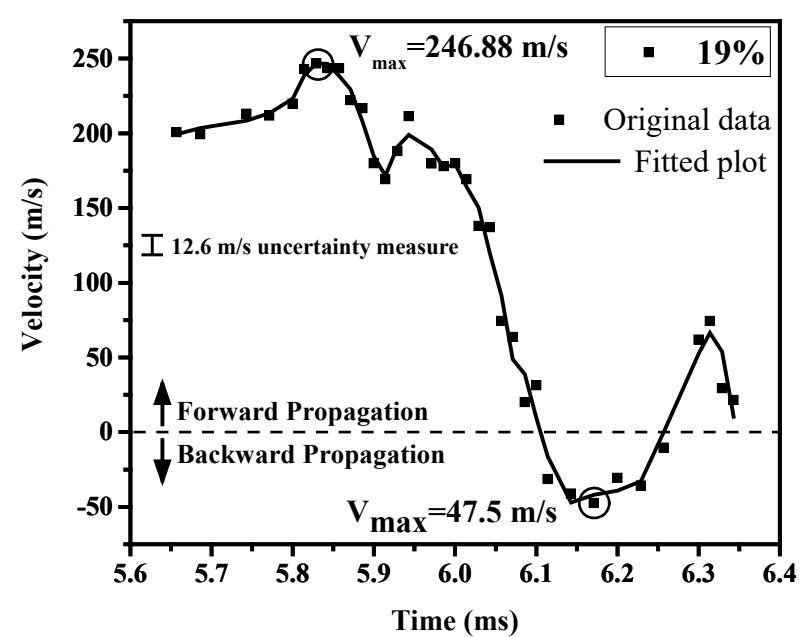

(a) Flame tip velocity versus time under an oxygen concentration of $19 \%$

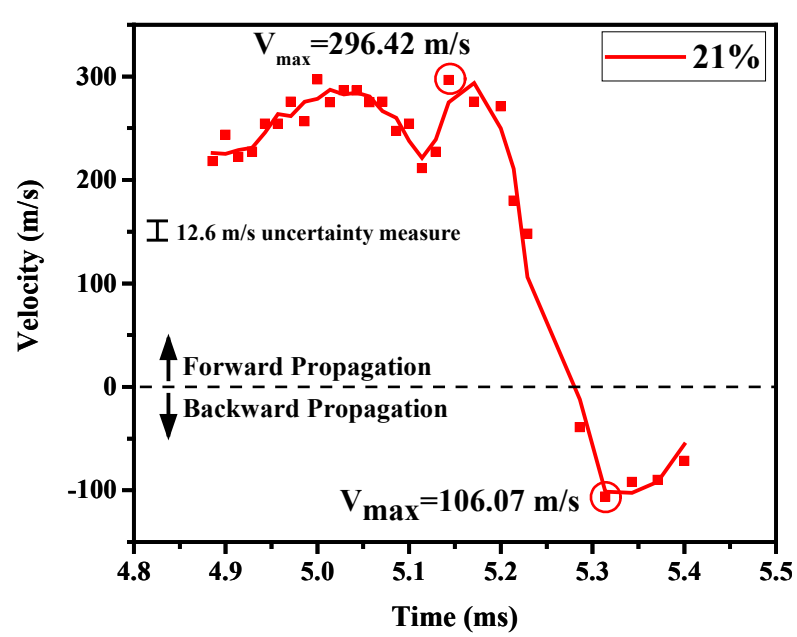

(b) Flame tip velocity versus time under an oxygen concentration of $21 \%$ 


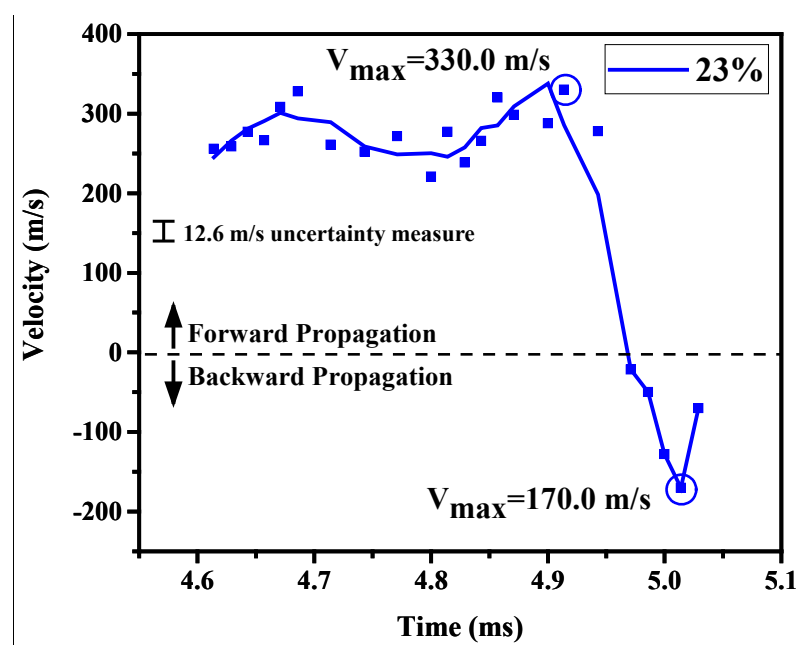

(c) Flame tip velocity versus time under an oxygen concentration of $23 \%$

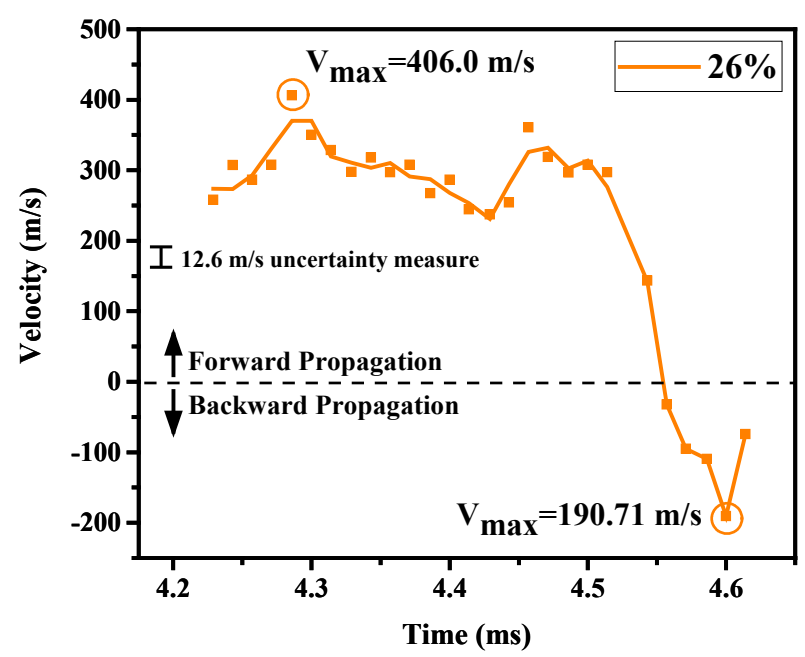

(e) Flame tip velocity versus time under an oxygen concentration of $26 \%$

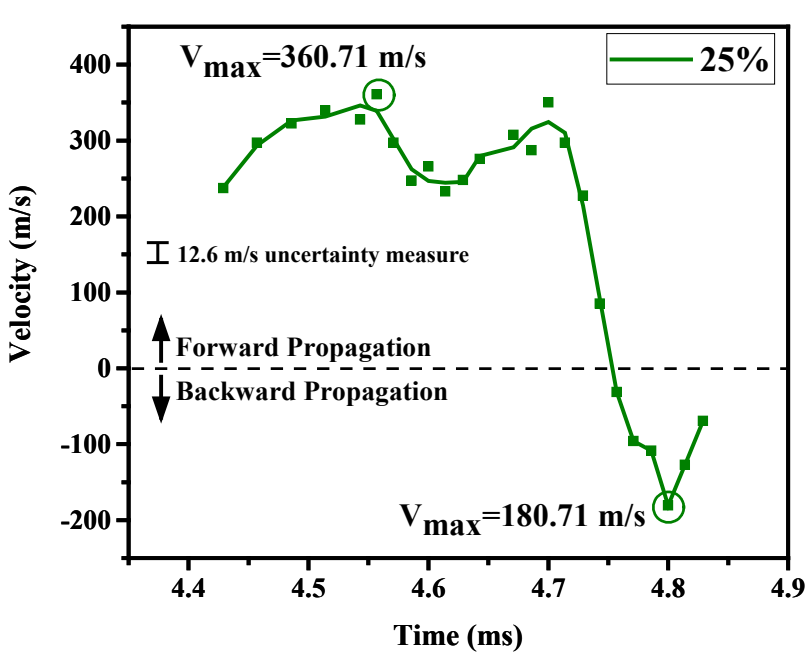

(d) Flame tip velocity versus time under an oxygen concentration of $25 \%$

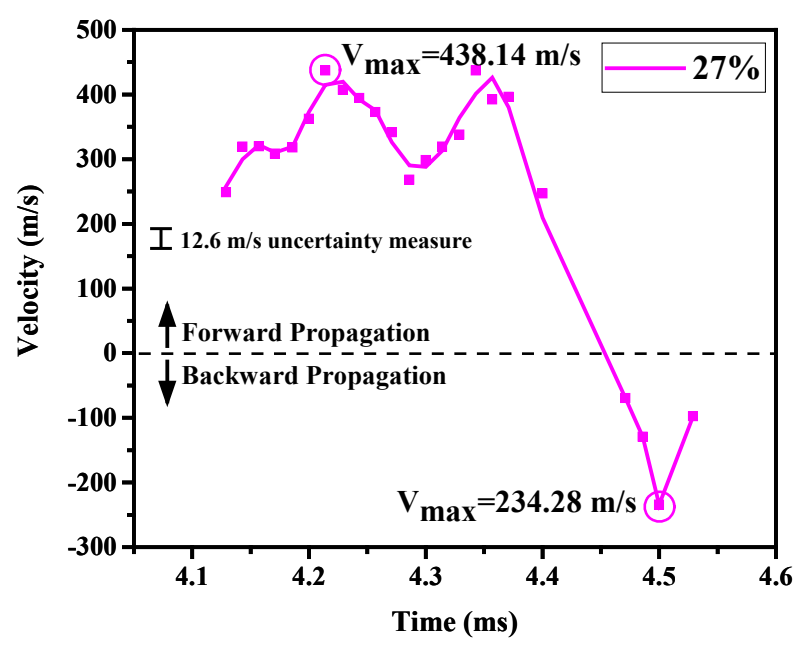

(f) Flame tip velocity versus time under an oxygen concentration of $27 \%$

Fig. 7 Turbulent flame tip velocity versus time behind the perforated plate under different oxygen concentrations

A comparison of the results in Figs. 5 and 7 reveals that, after the flame passes through the perforated plate, the flame tip velocity increases by an order of magnitude (approximately 10-100 m/s). Flame acceleration in obstructed channels has attracted great attention in combustion science. In [14], the authors proposed that delayed burning between the obstacles creates a powerful jet flow that drives the acceleration. With the burnt gas entering the holes of the perforated plate, the flow changes its direction to an axial one. Thus, it accumulates into a strong jet and pushes the leading flame tip forward. Apart from the influence of the powerful jet flow, in this study, a spherical flame was divided into several jet flames by the perforated plate. Hence, the Kelvin-Helmholtz and Rayleigh-Taylor instabilities also play an important role in increasing the flame surface area and the flame burning 
velocity. As illustrated in Fig. 7, after the flame passes through the perforated plate, the variation of the flame tip velocity under different oxygen concentrations presents a consistent trend: the flame tip velocity increases initially, followed by a decrease, then increases again, and finally decreases. The initial acceleration occurs because the flame is still promoted by powerful jet flows, which entrain the flame through the perforated plate. Later, as the powerful jet flows depart from the perforated plate, the flow velocity naturally slows down, which consequently cause the flame to decelerate. However, after the deceleration, the flame tip velocity increases again. In this process, the turbulent flame performs self-similar and self-acceleration processes, which resemble the "finger flame" acceleration mechanism [41, 42]. Finally, the flame tip velocity decreases and even becomes negative, which is caused by the reflected pressure/shock waves. It is noted that after the spark ignition, the flame propagates from left to right as illustrated in Fig. 1 (b). In Fig. 7, the negative flame tip velocity represents a propagation from right to left referring to a fixed coordinate in the laboratory frame. Once the pressure/shock waves reach the perforated plate, they will be reflected back and propagate towards the flame. As a result, the reflected pressure/shock waves reverse the flow of gas in front of the propagating flame, which consequently causes the flame tip velocity to decrease and even reverse. Actually, the flame propagation in the combustion chamber is influenced by various factors, including flame instabilities, flow field and pressure waves. In [30], the underlying mechanism of the flame acceleration and evolution of the flame tip velocity were discussed in detail.

As illustrated in Fig. 7, the overall flame tip velocity probably increases with increasing oxygen concentration. The maximum flame tip velocity is positively correlated with the oxygen concentration. As illustrated in Fig. 5, the increased oxygen concentration promotes the flame evolution before the perforated plate and consequently facilitates turbulent flame propagation behind the perforated plate. It is well known that shock waves are formed by the coalescence of compression waves, which are produced by the piston effect associated with the advancing flame [43]. More discussions about the formation and enhancement of shock waves can be found in our previous work [30]. It was found that faster flame tip velocity compresses the unburnt mixture more strongly, thereby inducing a stronger shock wave (Fig. 6) and promoting the end-gas autoignition. In the end region, (inhibited by the leftgoing reflected acoustic/shock wave) the flame decelerates and even countermarches. Thus, the maximum backward flame tip velocity is also positively correlated with the oxygen concentration. As discussed before, a higher oxygen concentration induces stronger shock waves. Therefore, after the 
shock wave is reflected at the end wall, it imposes a stronger inhibiting effect on the flame front, thereby causing a higher backward flame tip velocity.

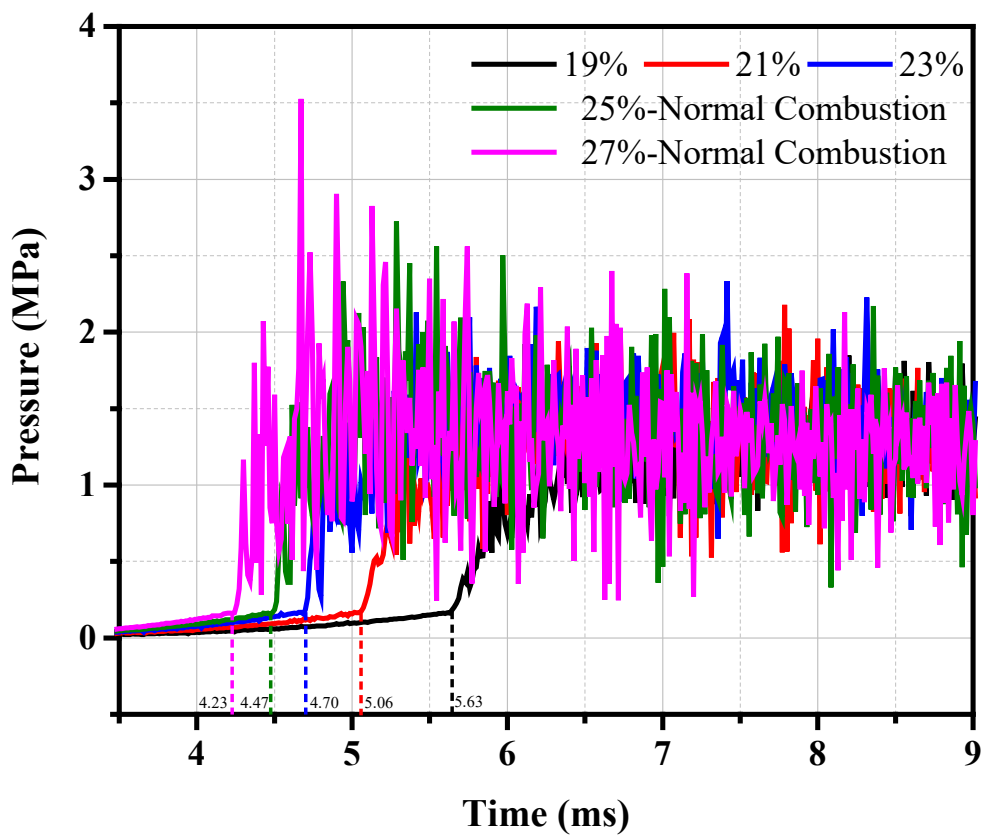

Fig. 8 Time history of the in-cylinder pressure under different oxygen concentrations
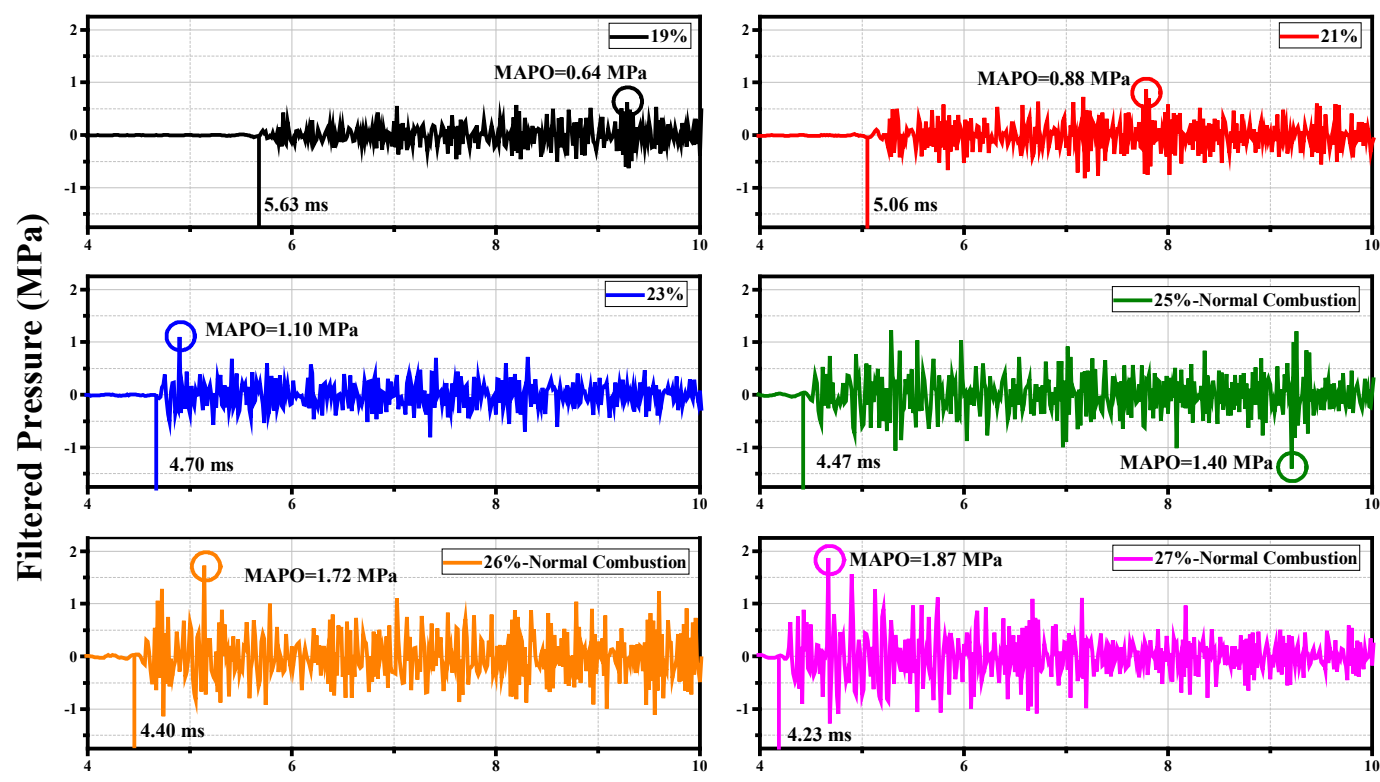

Time (ms)

Fig. 9 Pressure oscillation (filtered by a $4 \mathrm{kHz}$ high-pass filter) under different oxygen concentrations 


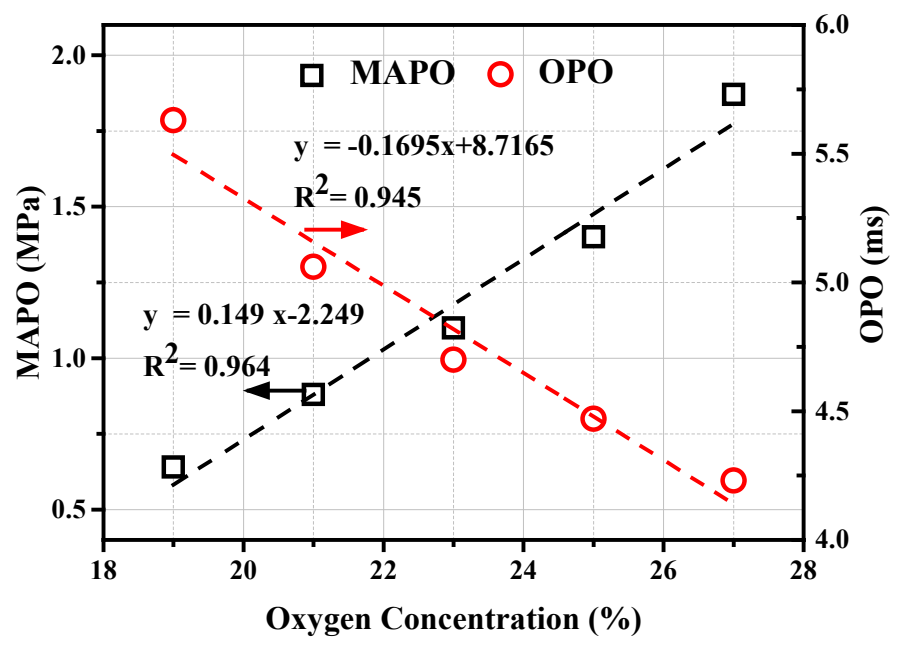

Fig. 10 Comparison of maximum amplitude of pressure oscillation (MAPO) and onset of pressure oscillation (OPO) under different oxygen concentrations

In this part, the effects of the oxygen concentration on the pressure and pressure oscillation are discussed. The original pressure data obtained with the pressure transducer (Kistler 6045A) under different oxygen concentrations are illustrated in Fig. 8. Fig. 9 illustrates the pressure oscillation obtained with a $4 \mathrm{kHz}$ high-pass filter. In addition, two important parameters, the maximum amplitude of the pressure oscillation (MAPO) and the onset of the pressure oscillation (OPO), are summarized and illustrated in Fig. 10. As illustrated in Fig. 8, intense pressure oscillation is observed. According to [44], the flame-sound interaction strongly influences intrinsic oscillations of a flame front and pressure. Owing to the intense pressure oscillation in Fig. 8, the lower pressure trace is covered by the higher one. To avoid further overlaps, the pressure trace obtained with an oxygen concentration of $26 \%$ is not presented. The peak pressure increases with increasing oxygen concentration. In addition, as illustrated in Fig. 9, the MAPO is positively correlated with the oxygen concentration. As discussed in Fig. 6, the intensity of the shock waveincreases with increasing oxygen concentration, thereby increasing the pressure and pressure oscillation. Moreover, the OPO shifts to an earlier time as the oxygen concentration increases. Figure 10 provides a visual representation of the relationships of the MAPO and OPO with the oxygen concentration. The MAPO and OPO are approximately linearly related to the oxygen concentration. The liner function was acquired via the least-squares method. For MAPO, the function is $\mathrm{y}=0.149 \mathrm{x}-2.249$ and the coefficient is $\mathrm{R}^{2}=0.964$. For OPO, the function is $\mathrm{y}=-0.1695 \mathrm{x}+8.7165$ and the coefficient is $\mathrm{R}^{2}=0.945$.

\subsection{End-gas autoignition with detonation}

In the previous part, the effects of the oxygen concentration on the flame and shock waves propagation, pressure, and pressure oscillation without end-gas autoignition are discussed. As 
illustrated in Fig. 2, end-gas autoignition can occur when the oxygen concentration increases to $25 \%$. In this section, the end-gas autoignition is analyzed. Figure 11 illustrates the sequential Schlieren images of an end-gas autoignition with detonation development. The original high-speed Schlieren images are provided as Supplemental Material (called "Autoignition Mode1" and "Autoignition Mode 2"). After the flame passes through the perforated plate, it accelerates sharply, which compresses the unburnt mixture and induces shock waves. As illustrated in Fig. 11, once the shock wave arrives at the end wall, it is reflected and propagates toward the left at $4.671 \mathrm{~ms}$. A mutual inhibition competition occurs between the reflected shock wave and the turbulent flame owing to their opposite propagation directions. Owing to the flame-shock wave interaction, the flame decelerates and even propagates backward, which provides more time for the end-gas autoignition. At $4.700 \mathrm{~ms}$, the reflected shock wave enters the burnt region. Note that a shock wave propagating in a burnt mixture is not as evident as one propagating in an unburnt mixture. This is because the density gradient across the shock wave in the burnt mixture is much smaller than that in the unburnt mixture [48]. Moreover, it is extremely difficult to capture shock waves in such turbulent flow fields in the burned gas region using Schlieren diagnostics. Once the shock wave approaches the perforated plate, it is reflected again and propagates to the right at $4.857 \mathrm{~ms}$. At $4.971 \mathrm{~ms}$, the shock wave reaches the end wall and experiences another reflection. Then, end-gas autoignition occurs due to the repeated compression of shock waves. It is noted that, owing to the limited image sample frequency in the present work, the origin of the hot spots can hardly be caught. Subsequently, a strong pressure wave with high luminosity propagates from right to left with a velocity of $1659.3 \mathrm{~m} / \mathrm{s}$. This is close to the Chapman-Jouguet (C-J) velocity (1993.30 $\mathrm{m} / \mathrm{s}$ ) obtained in a zero-dimension simulation under equal initial conditions. Therefore, the left-going strong pressure wave was confirmed to be a detonation wave, at least at the initial stage in the unburnt end-gas region, which gradually transforms into a shock wave in the burnt region. The detonation is the result of an autoignition at a hot spot, which is induced by the repeated compression of shock waves. The shock waves are sufficiently strong to induce a local, strong fresh-gas temperature increase, thereby leading to a substantial decrease in the autoignition delay. This enables coupling between the shock wave and autoignition reaction rate, which reinforces each other and leads to an extremely high pressure and propagation speed similar to those of a detonation. As discussed in [26], the coupling between the pressure wave and autoignition flame leads to a detonation combustion wave in the gasoline engine. 


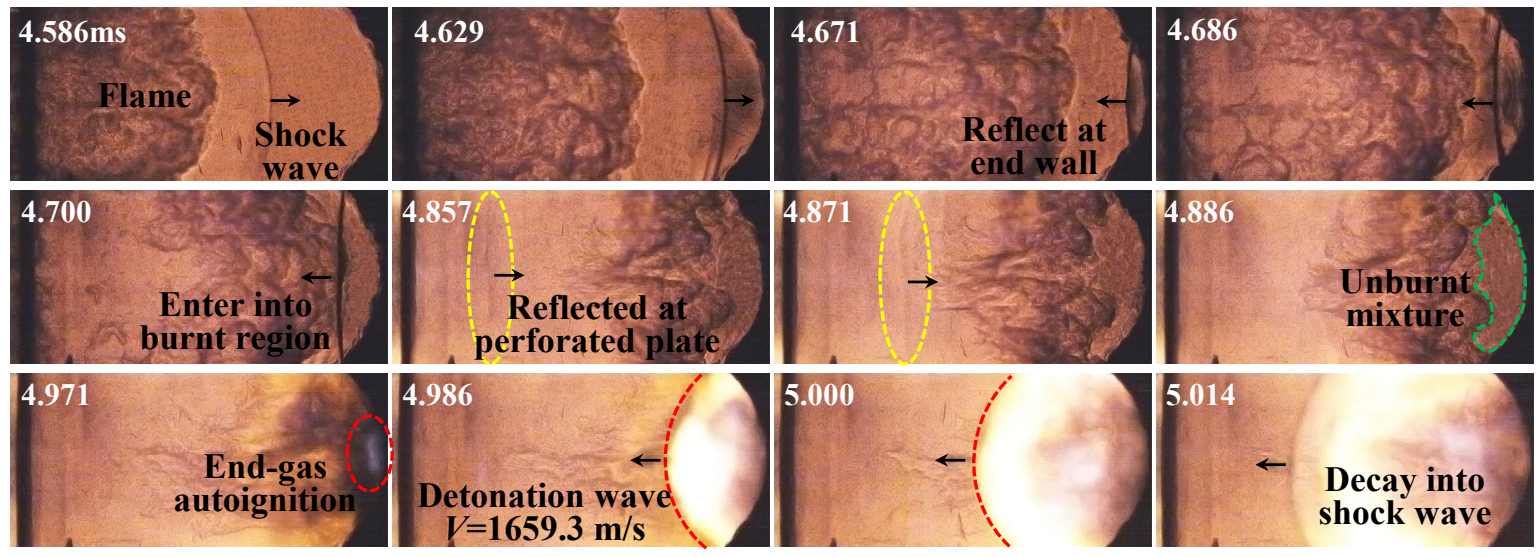

(a) Mode 1
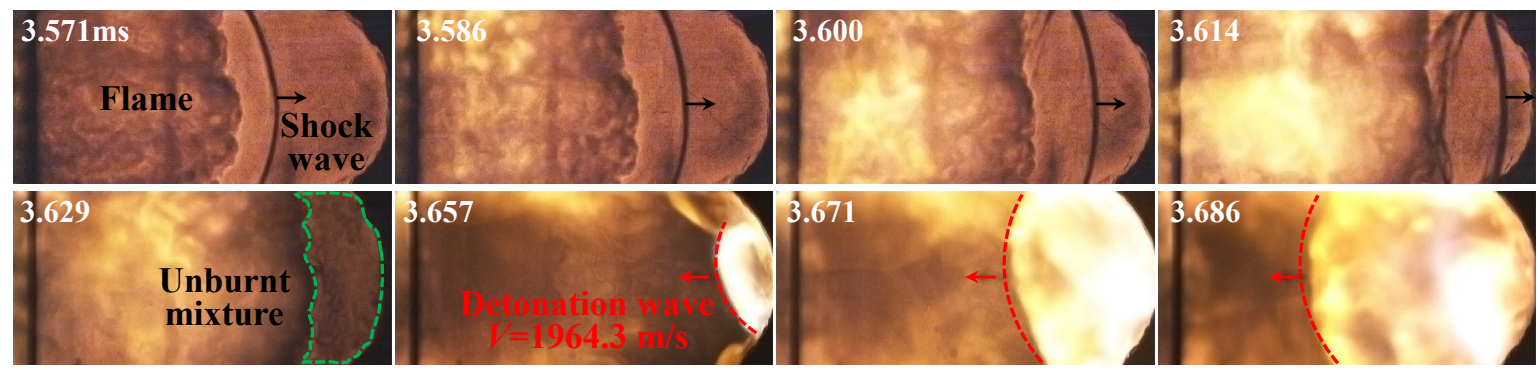

(b) Mode 2

Fig. 11 Sequential Schlieren images of two kinds of detonation initiation modes

In this study, two kinds of detonation modes were found. Based on the time of the shock wave reflection at the end wall before the autoignition, the detonation development is classified as Mode 1 or Mode 2. As illustrated in Fig. 11 (a), under detonation Mode 1, the end-gas autoignition occurs after the shock wave has been reflected two times at the end wall. However, under detonation Mode 2, the end gas suffers compression owing to the shock wave only once before the autoignition. Based on the Schlieren images, no more differences can be found between Mode 1 and Mode 2. Their pressures and pressure oscillations are compared in Fig. 12.

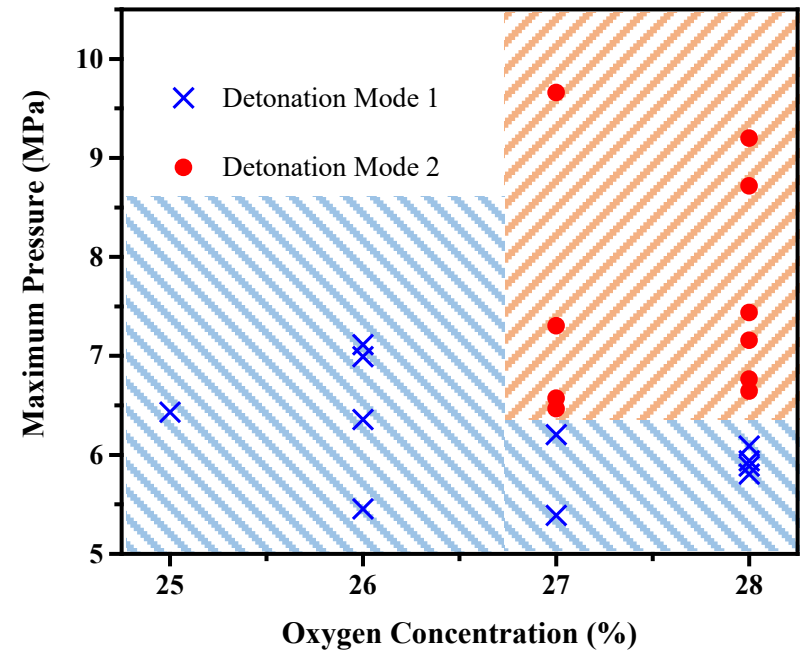

(a) Maximum pressure of detonation cases

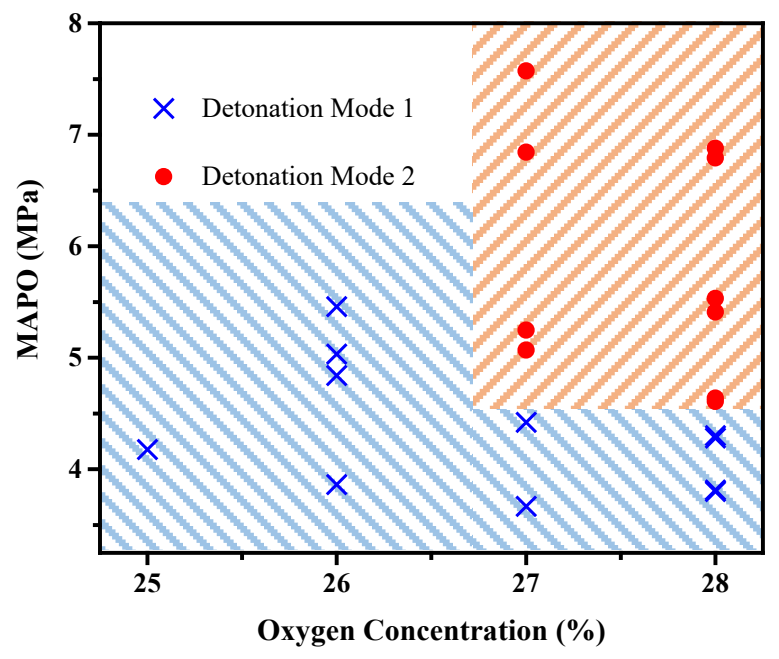

(b) MAPO of detonation cases

Fig. 12 Summary of maximum pressure and MAPO of detonation cases under different oxygen 


\section{concentrations}

As illustrated in Fig. 12, under oxygen concentrations of $25 \%$ and $26 \%$, only detonation Mode 1 exists. When the oxygen concentration increases to $27 \%$ and $28 \%$, detonation Mode 2 appears. As discussed before, the shock wave intensity increases with increasing oxygen concentration and generates stronger compression and heat effects on the unburnt mixture, which can provide more appropriate conditions for autoignition. Therefore, under higher oxygen concentrations, autoignition can occur after only one compression owing to the shock wave. In addition, the maximum pressure and MAPO of detonation Mode 2 are higher than those of detonation Mode 1 for equal oxygen concentrations. As discussed in [28], the knocking intensity is strongly related to the mass fraction of the unburnt mixture in real engines. A large amount of unburnt mixture generates a high pressure and strong pressure oscillation. In Fig. 11, the end unburnt mixture before the autoignition is marked by a region surrounded by a green dotted line. Under detonation Mode 2, larger amounts of unburnt mixture, which auto ignites, exist than under detonation Mode 1 before end-gas autoignition occurs. Thus, the pressure and pressure oscillation are higher under detonation Mode 2 than under detonation Mode 1.

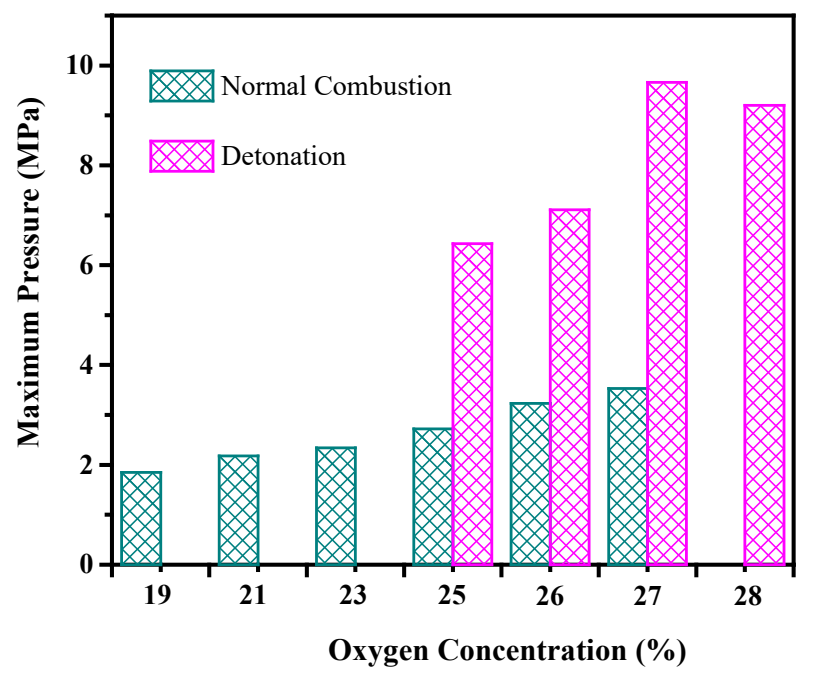

Fig. 13 Comparison of maximum pressures of normal combustion and end-gas autoignition with detonation development 

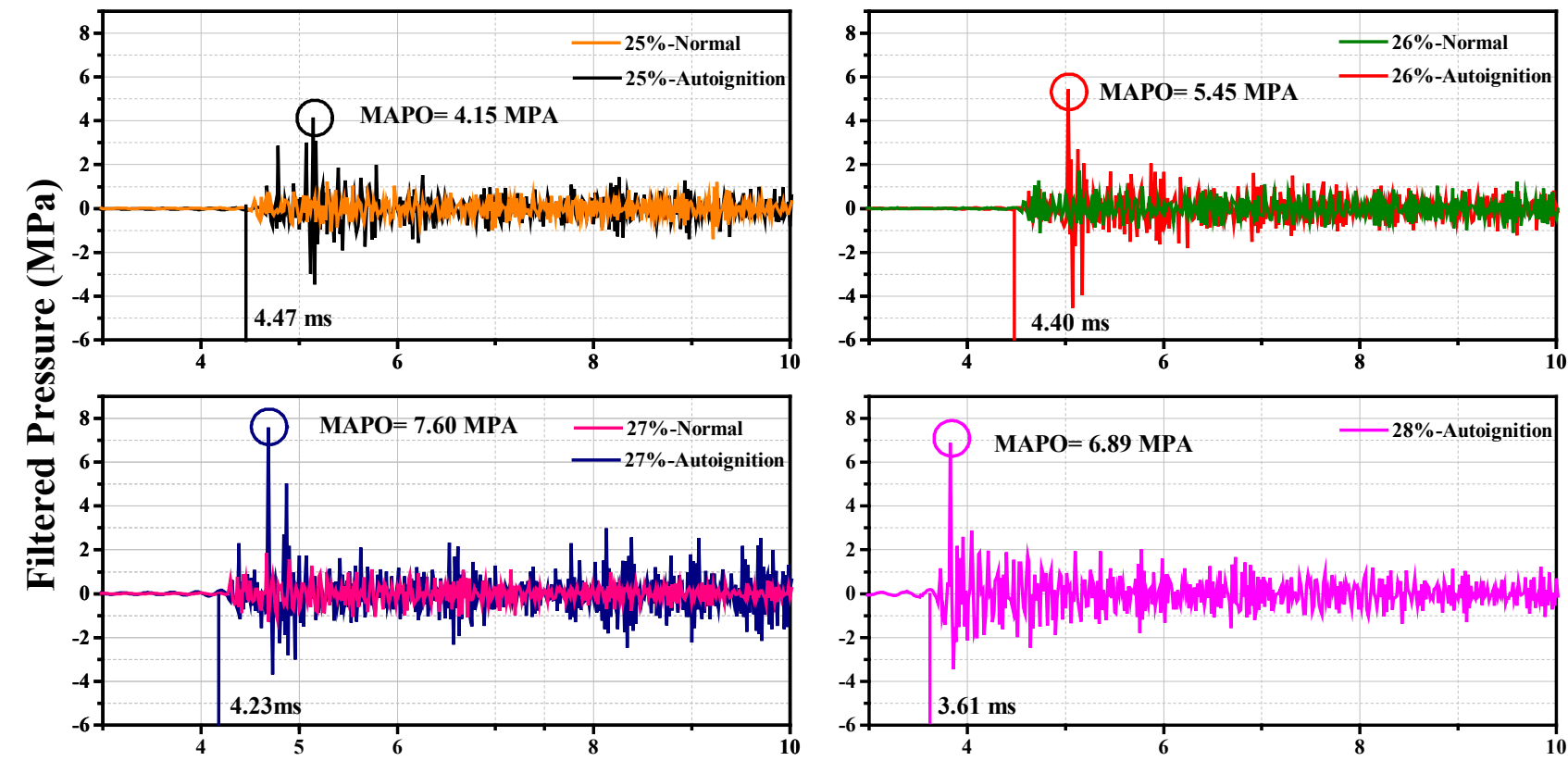

Time (ms)

\section{Fig. 14 Comparison of pressure oscillations of normal combustion and end-gas autoignition with detonation development}

In the following, the maximum pressure, pressure oscillation, and flame tip velocity of the normal combustion and end-gas autoignition are compared. The comparisons of the maximum pressure and pressure oscillation are illustrated in Figs. 13 and 14, respectively. The maximum pressure of the selected cases is approximately equal to the mean pressure of all cases. As illustrated in Fig. 13, the maximum pressure of the end-gas autoignition is much higher than that of the normal combustion. In this study, the end-gas autoignition was always accompanied by detonation development. Once the detonation occurs, the end unburnt mixture is consumed in a very short time, which induces an extremely high pressure. In [10], Benedetto attributed the very high pressure peaks to the occurrence of a so-called "combustion-induced rapid phase transition". The $4 \mathrm{kHz}$ high-pass filtered pressure is illustrated in Fig. 14. Under equal oxygen concentrations, the OPO values of the normal combustion and end-gas autoignition remain equal. Thus, whether or not an end-gas autoignition occurs is not directly related to the initial combustion development stage. However, owing to the strong and fast detonation wave, the pressure oscillation with end-gas autoignition is extremely high. Under an oxygen concentration of $27 \%$, the MAPO with end-gas autoignition is $7.60 \mathrm{MPa}$, which is approximately four times higher than that of the normal combustion. Once the end-gas autoignition occurs, it induces an extremely high pressure and strong pressure oscillations. However, the pressures and pressure oscillations of the end-gas autoignition are found to be random and essentially unrelated to the oxygen concentration (Fig. 12) 


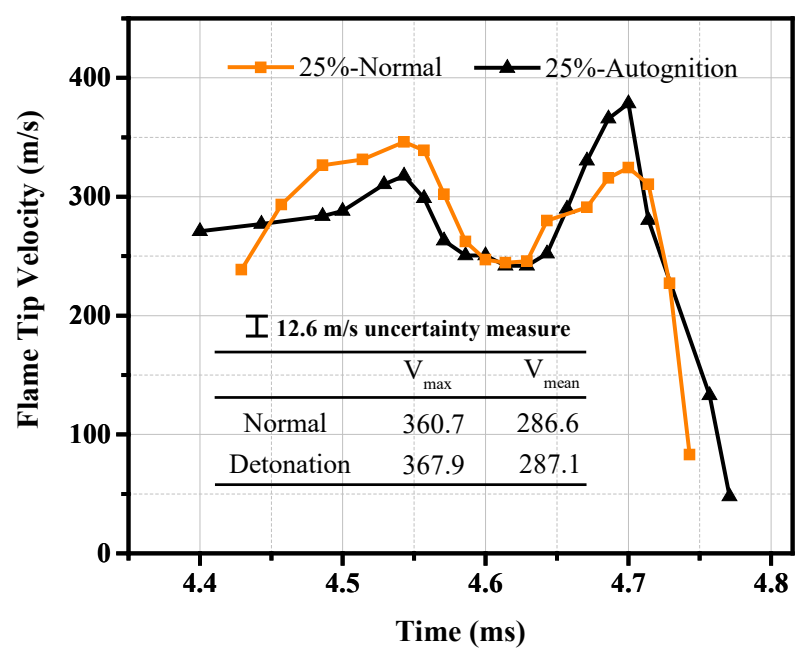

(a)

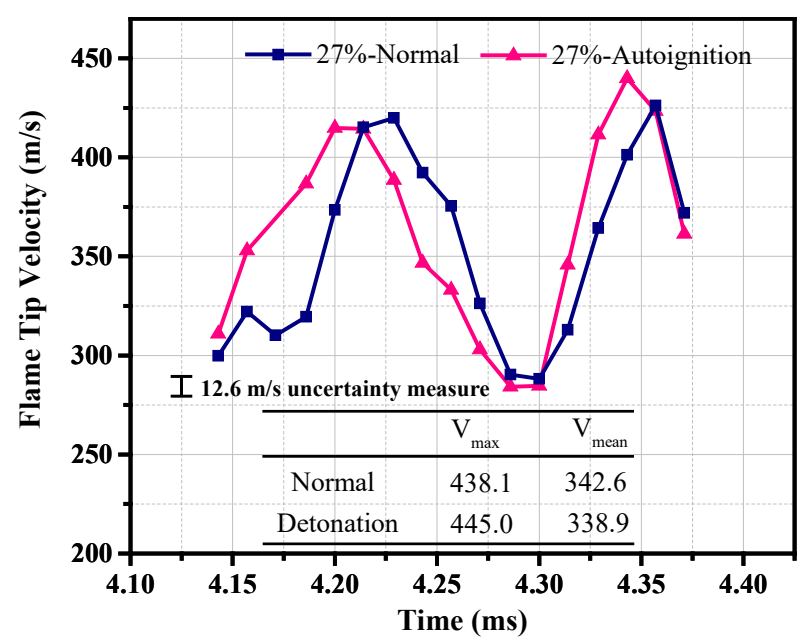

(c)

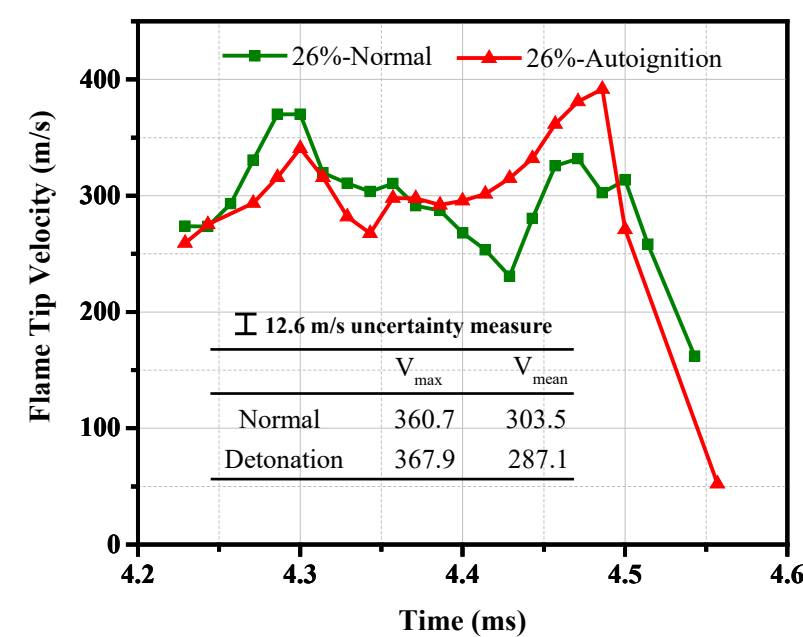

(b)

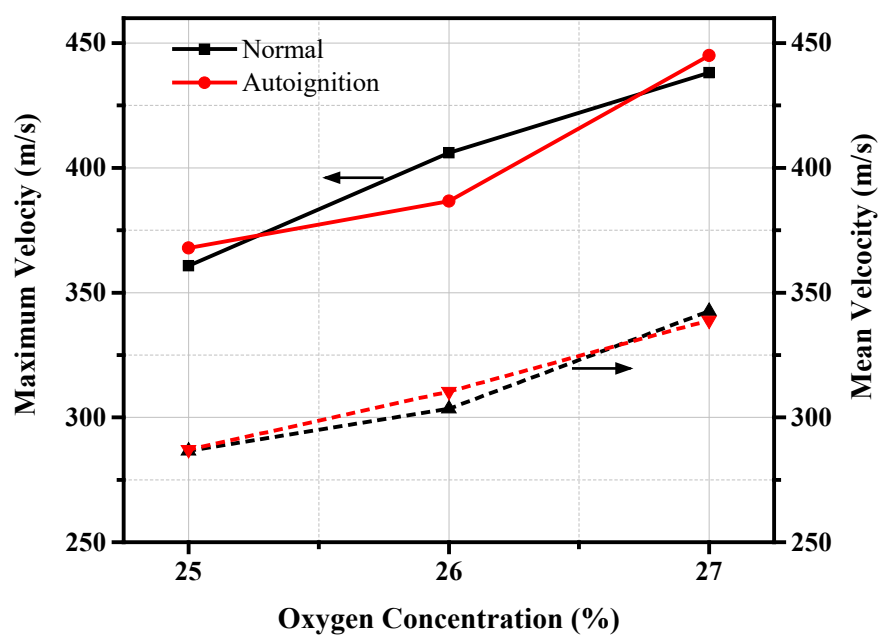

(d)

Fig. 15 Comparison of flame tip velocities of normal combustion and end-gas autoignition with detonation development

As shown in Fig. 7, with the increasing oxygen concentration, the flame tip velocity after the perforated plate increases, which will induce stronger shock waves shown in Fig. 6 and consequently promote the end-gas autoignition. Figure 15 compares the flame tip velocities of the normal combustion and end-gas autoignition under the same oxygen concentrations. The flame tip velocity remains approximately constant in term of development tendency and value. The maximum velocity and mean velocity are illustrated in Fig. 15 (d). The mean velocity of the normal combustion exhibits approximately no differences with end-gas autoignition. Thus, combining Fig. 7 and 15, it concluded that the high flame velocity is necessary but not sufficient for end-gas autoignition. As previously mentioned, the DDT is a stochastic and sensitive process. The slight difference in flame tip velocities between normal combustion and end-gas autoignition further validates the stochastic nature of endgas autoignition in a certain condition. The small fluctuations of the flame tip velocity might cause a series of chain reactions, which finally lead to a different combustion phenomenon. It is worth stressing 
that end-gas autoignition with detonation development is a complex physical system, which depends on multiple phenomena, including flow instabilities; turbulence; many interactions between shocks, flames and vortices; and a resulting hot-spot formation. Therefore, to predict an end-gas autoignition occurrence, more fundamental data on the flow dynamics, turbulence and chemical kinetics are necessary.

\subsection{Effects of exhaust gas}

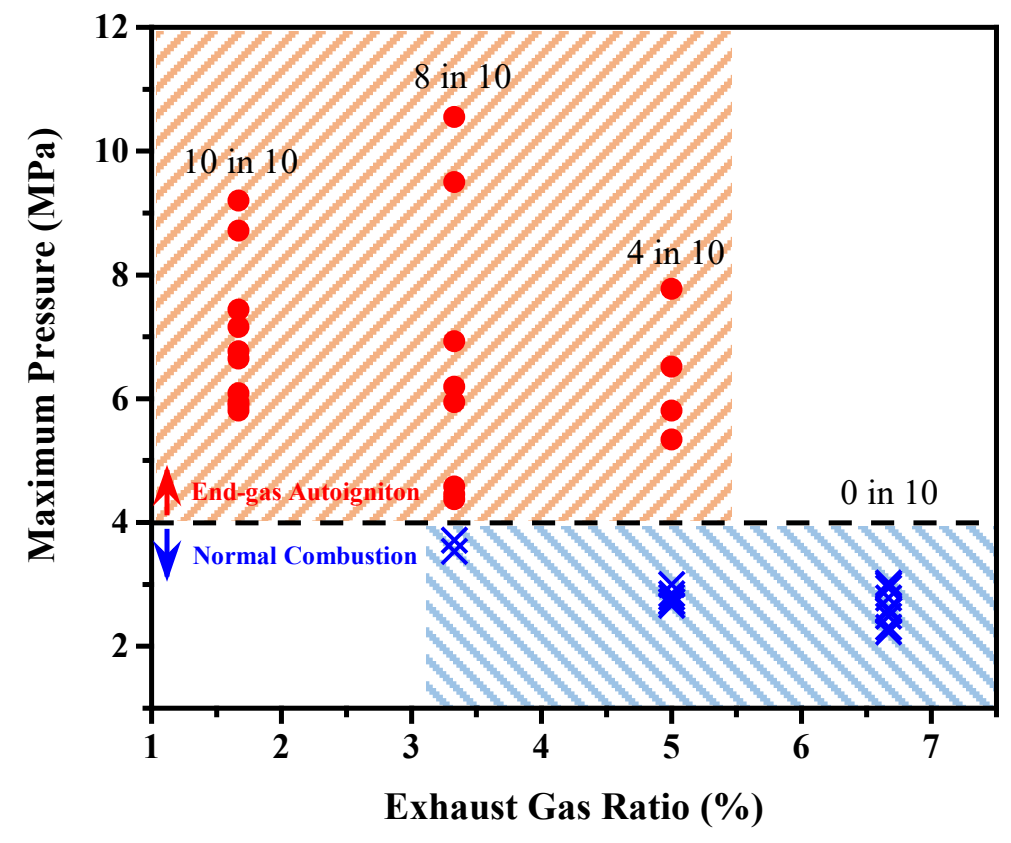

Fig. 16 Maximum pressure under different exhaust gas ratios

As previously discussed, with increasing oxygen and decreasing nitrogen, the probability of endgas autoignition occurrence with detonation development increases. Significant advances [45-50] have been made over the past years in the understanding of the influence of diluted inert gas on the detonation and flame propagation. In this study, exhaust gas (consisting of water vapor and nitrogen) was introduced into the initial unburnt mixture before the spark ignition to optically investigate the effects of the exhaust gas on the probability of end-gas autoignition occurrence. Exhaust gases such as $\mathrm{CO}_{2}$ and $\mathrm{H}_{2} \mathrm{O}$ are generally employed to suppress the knock occurrence [17]. In this study, the exhaust gas ratio (EG ratio) was calculated with Eq. (3), and four cases were chosen (EG ratio $=1.67 \%, 3.33 \%$, $5 \%$, and $6.67 \%$ ). The experiments were repeated ten times for every exhaust gas ratio. It was observed that the combustion chamber cannot be evacuated completely. Thus, the smallest exhaust gas ratio was set to $1.67 \%$. The oxygen concentration was $28 \%$ in all experiments.

$$
\text { EG ratio }=\frac{P_{\text {exhaust gas }}}{P_{\text {total }}}
$$

Where $P_{\text {exhaust gas }}$ is the partial pressure of the exhaust gas, and $P_{\text {total }}$ is the total pressure. 
The maximum pressures under different exhaust gas ratios are illustrated in Fig. 16. With increasing exhaust gas ratio, the probability of end-gas autoignition occurrence decreases; it occurs 10 out of 10 times with an exhaust gas ratio of $1.67 \%, 8$ out of 10 times with an exhaust gas ratio of $3.33 \%, 4$ out of 10 times with an exhaust gas ratio of $5 \%$, and 0 out of 10 times with an exhaust gas ratio of $6.67 \%$. The exhaust gas is composed of $\mathrm{H}_{2} \mathrm{O}$ and $\mathrm{N}_{2}$, which have retardant effects on the combustion. Water and nitrogen have high specific heat capacities, and consequently decrease the burning temperature. In addition, the introduced exhaust gas dilutes the hydrogen-oxygen mixture; thus, retarding the chemical reaction. Therefore, the introduction of the exhaust gas hinders the occurrence of an end-gas autoignition. As illustrated in Fig. 16, the maximum pressure of the normal combustion decreases with increasing exhaust gas ratio. However, once an end-gas autoignition occurs, the maximum pressure is stochastic and seems to be unrelated to the exhaust gas ratio. As discussed before, the maximum pressure is related to the amount of reactive gas consumed at once through the autoignition process. However, the start time of end-gas autoignition is stochastic, causing the amount of reactive gas which auto ignites random. Thus, the maximum pressure with end-gas autoignition is stochastic.
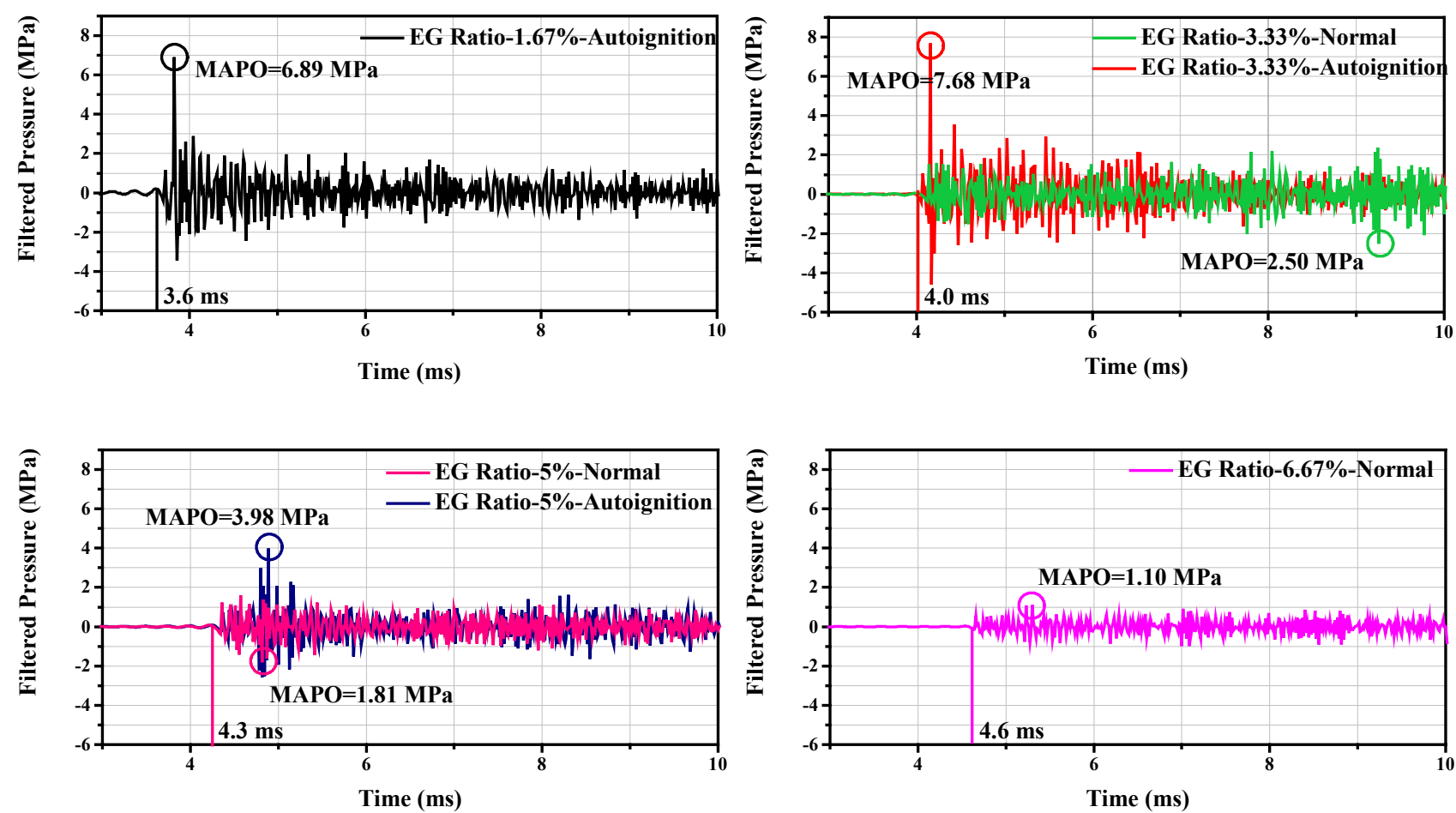

Fig. 17 Filtered pressure (4 kHz high-pass filter) under different exhaust gas ratios

The filtered pressure obtained with the $4 \mathrm{kHz}$ high-pass filter under different exhaust gas ratios is illustrated in Fig. 17. The maximum pressures are approximately equal to the respective mean value of the maximum pressures. Under equal exhaust gas ratios, the pressure oscillation with end-gas 
autoignition is much higher than that of the normal combustion. It should be noted that the MAPO of the normal combustion decreases with increasing exhaust gas ratio. This is because the introduction of exhaust gas dilutes the hydrogen-oxygen mixture and inhibits the chemical reaction. The similar results were conducted experimentally by Wang et al. [46] regarding detonation propagations in a stoichiometric $\mathrm{H}_{2}-\mathrm{O}_{2}$ mixture diluted with $\mathrm{Ar}$ and $\mathrm{N}_{2}$. They pointed out that the velocity decreases and the limiting pressure increases with increasing dilution concentration. This indicates that the detonation propagation ability is weakened with the addition of the inert gases, in particular $\mathrm{N}_{2}$.

The evolution of the flame tip velocity under different exhaust gas ratios is illustrated in Fig. 18. As illustrated in Fig. 7, after the flame passes through the perforated plate, the variation tendencies of the flame tip velocity under different exhaust gas ratios exhibit a similar trend: the flame tip velocity increases initially, followed by a decreases, then increases again, and finally decreases, forming an "M" shape. The impact factors regarding the flame propagation have already been discussed in Fig. 7. As illustrated in Fig. 18, the maximum flame tip velocity decreases with increasing exhaust gas ratio. According to Valiev et al. [42], higher flame tip velocity could strengthen the compression wave (disturbance flow behind shock wave) stronger. In our previous work [30,31], it is found that intense shock/compression waves can result in strong pressure oscillations, explaining the consistency between the pressure oscillations and the flame tip velocity. The introduction of water and nitrogen mainly has two general effects on the chemical reaction: (1) a dilution effect, which lowers the concentration of the reactive species, and (2) a thermal effect due to the change in the burning temperature. Firstly, the exhaust gas hinders the molecular collision of hydrogen and oxygen, which retards the chemical reaction. Secondly, the addition of inert gases decrease total specific heat release, since the inert gases absorb some of the energy during chemical reaction, which slows down the chemical reaction. Both aspects are responsible for the decrease in the pressure and pressure oscillation with increasing exhaust gas ratio. 


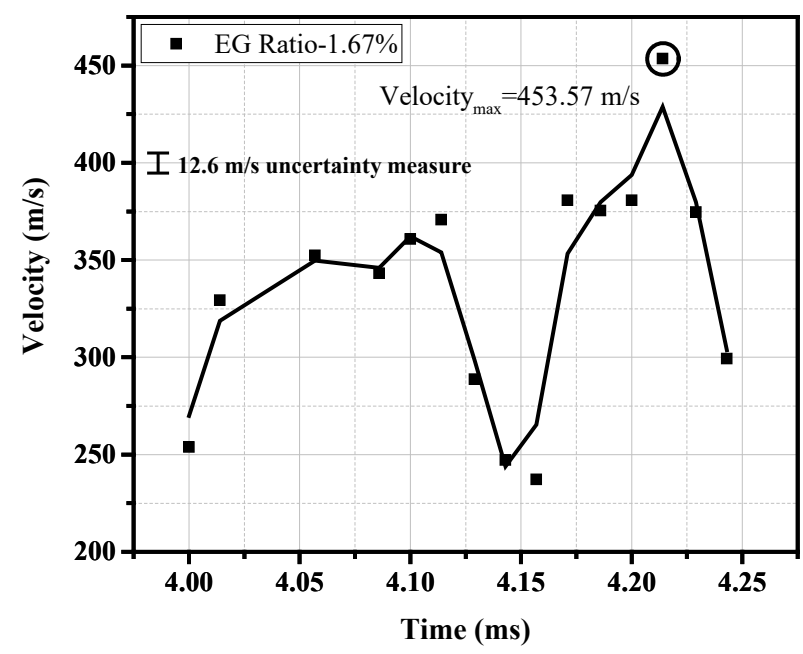

(a)

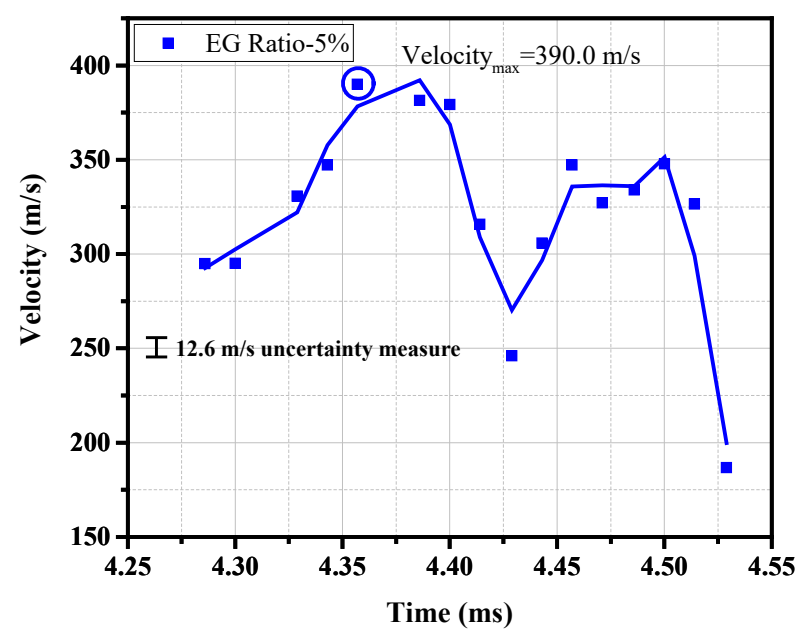

(c)

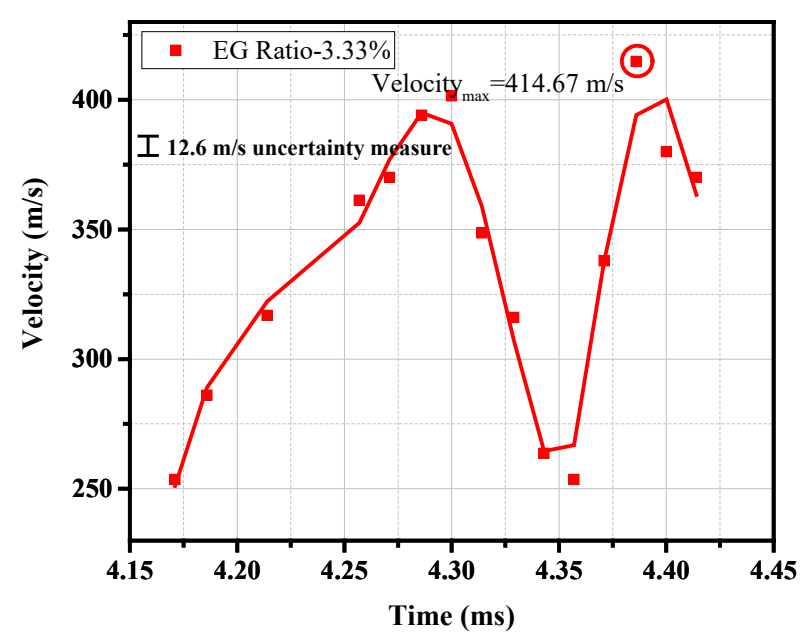

(b)

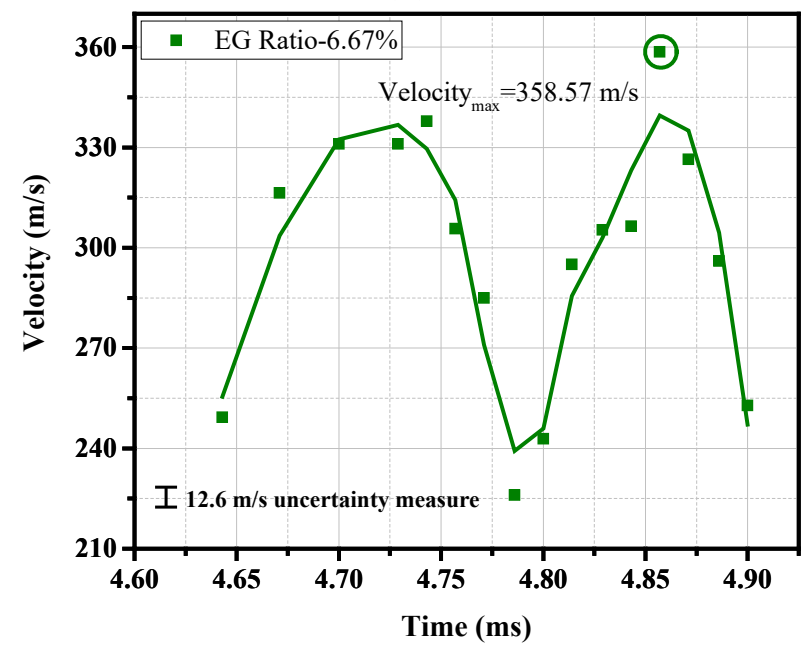

(d)

Fig. 18 Flame tip velocity under different exhaust gas ratios

\section{Conclusion}

Experiments were conducted in a self-designed CVCB to investigate the effects of oxygen concentration on the flame and shock wave propagations, pressure, and pressure oscillation. Under an oxygen-enriched condition, the probability of end-gas autoignition occurrence increases substantially. Further, end-gas autoignition with detonation development with a special emphasis on the stochasticity of DDT was investigated. Exhaust gas was introduced into the initial unburnt mixture to investigate the effect of an inert gas on the combustion. Four main conclusions can be drawn:

(1). With an oxygen concentration increasing from $19 \%$ to $28 \%$, the probability of end-gas autoignition with detonation development occurrence increases. Under an oxygen concentration of $25 \%$, the endgas autoignition starts to appear with limited repetitions. When the oxygen concentration increases to $28 \%$, end-gas autoignition always occurs. Under low oxygen concentrations, the detonation is random owing to its stochastic flow instabilities, turbulence, and hot-spot formation. Nevertheless, as the 
oxygen concentration increases, the stochastic factors play minor roles, and the detonation becomes a certain event.

(2). The variation tendency of the flame tip velocity remains constant under different oxygen concentrations. Before the perforated plate, the flame tip velocity initially increases and then decreases. After passing through the perforated plate, the curve of the turbulent flame tip velocity exhibits an "M" shape. The increasing oxygen concentration is beneficial to the reaction rate and consequently improves the flame tip velocity. The faster flame tip velocity under higher oxygen concentrations results in a stronger compression of the unburnt mixture, which induces a stronger shock wave and consequently promotes the end-gas autoignition. With increasing oxygen concentration, the pressure and pressure oscillation of the normal combustion increase. In addition, the MAPO and OPO are linearly correlated with the oxygen concentration. However, once the end-gas autoignition occurs, the pressure and pressure oscillation exhibit random behaviors owing to the stochastic nature of a DDT.

(3). Based on the start time of the end-gas autoignition, two kinds of end-gas autoignition modes could be observed. Under detonation Mode 1, the autoignition occurs after two shock wave reflections at the end wall. Under detonation Mode 2, the end gas experiences only one compression by the shock wave before the autoignition. Further, Mode 2 only occurs under high oxygen concentrations of $27 \%$ and $28 \%$. Under equal oxygen concentrations, the pressure and pressure oscillation of Mode 2 are higher than those of Mode 1 owing to the larger amount of unburnt mixture.

(4). Exhaust gas was introduced to the initial unburnt mixture to investigate the effect of the inert gas on the combustion. With increasing exhaust gas ratio, the end-gas autoignition occurrence probability, flame tip velocity, and pressure oscillation decrease, due to the diluting effect and high specific heat capacity of the exhaust gas.

Further studies on practical situations with realistic fuel properties, thermodynamic conditions, and flow characteristics are necessary for the thorough understanding of knock/super-knock combustion in real engines. In addition, more fundamental data of the flow dynamics and chemical kinetics must be obtained through advanced measurement technologies for a better understanding of end-gas autoignition and detonation development in confined spaces.

\section{Acknowledgment}

This work was supported by the National Science Fund for Distinguished Young Scholars (Grant No. 
51825603) and the National Natural Science Foundation of China (Grant No. 91741119, 51606133).

This paper is supported by the opening project of State Key Laboratory of Explosion Science and Technology (Beijing Institute of Technology). The opening project number is KFJJ18-09M.

\section{References}

[1] C.K. Law, G. Jomaas, J.K. Bechtold, Cellular instabilities of expanding hydrogen/propane spherical flames at elevated pressures: theory and experiment, P Combust Inst 30 (2005) 159-167.

[2] E.S. Oran, V.N. Gamezo, Origins of the deflagration-to-detonation transition in gas-phase combustion, Combust.Flame 148 (2007) 4-47.

[3] A. Misdariis, O. Vermorel, T. Poinsot, LES of knocking in engines using dual heat transfer and two-step reduced schemes, Combust.Flame 162 (2015) 4304-4312.

[4] D. Valiev, V. Bychkov, V.y. Akkerman, C.K. Law, L.-E. Eriksson, Flame acceleration in channels with obstacles in the deflagration-to-detonation transition, Combust.Flame 157 (2010) 1012-1021.

[5] T. Poinsot, D. Veynante, Theoretical and Numerical Combustion, R.t.edwards Inc, (2005).

[6] W. Han, Y. Gao, C.K. Law, Flame acceleration and deflagration-to-detonation transition in micro and macro-channels: An integrated mechanistic study, Combust.Flame 176 (2017) 285-298.

[7] V.N. Kurdyumov, M. Matalon, Self-accelerating flames in long narrow open channels, P Combust Inst 35 (2015) 921928.

[8] V.N. Gamezo, A.M. Khokhlov, E.S. Oran, The influence of shock bifurcations on shock-flame interactions and DDT, Combust.Flame 126 (2001) 1810-1826.

[9] V. Bychkov, D. Valiev, L.-E. Eriksson, Physical Mechanism of Ultrafast Flame Acceleration, Phys Rev Lett 101 (2008). [10] A. Di Benedetto, F. Cammarota, V. Di Sarli, E. Salzano, G. Russo, Anomalous behavior during explosions of CH4 in oxygen-enriched air, Combust.Flame 158 (2011) 2214-2219.

[11] H. Qin, J.H.S. Lee, Z. Wang, F. Zhuang, An experimental study on the onset processes of detonation waves downstream of a perforated plate, P Combust Inst 35 (2015) 1973-1979.

[12] S.B. Dorofeev, Flame acceleration and explosion safety applications, P Combust Inst 33 (2011) 2161-2175.

[13] G.D. Roy, S.M. Frolov, A.A. Borisov, D.W. Netzer, Pulse detonation propulsion: challenges, current status, and future perspective, Prog Energ Combust 30 (2004) 545-672.

[14] K.I. Shelkin, On Combustion in a Turbulent Flow, Technical Report Archive \& Image Library, (1947).

[15] G. Ciccarelli, S. Dorofeev, Flame acceleration and transition to detonation in ducts, Prog Energ Combust 34 (2008) 499-550.

[16] R.K. J.H.S. Lee, A. Freiman, High speed turbulent deflagrations and transition to detonation in H 2 ワair mixtures, Combust.Flame 56 (1984) 227-339.

[17] Z. Wang, H. Liu, R.D. Reitz, Knocking combustion in spark-ignition engines, Prog Energ Combust 61 (2017) 78-112.

[18] H. Yu, Z. Chen, End-gas autoignition and detonation development in a closed chamber, Combust.Flame 162 (2015) 4102-4111.

[19] G.A. Lavoie, J. Martz, M. Wooldridge, D. Assanis, A multi-mode combustion diagram for spark assisted compression ignition, Combust.Flame 157 (2010) 1106-1110.

[20] C.K. Westbrook, M. Mehl, W.J. Pitz, M. Sjoberg, Chemical kinetics of octane sensitivity in a spark-ignition engine, Combust.Flame 175 (2017) 2-15.

[21] Y. Zeldovich, Flame propagation in a substance reacting at initial temperature, Combust.Flame 39 (1980) 219-224.

[22] L. Bates, D. Bradley, G. Paczko, N. Peters, Engine hot spots: Modes of auto-ignition and reaction propagation, Combust.Flame 166 (2016) 80-85. 
[23] D. Bradley, G.T. Kalghatgi, Influence of autoignition delay time characteristics of different fuels on pressure waves and knock in reciprocating engines, Combust.Flame 156 (2009) 2307-2318.

[24] X.J. Gu, D.R. Emerson, D. Bradley, Modes of reaction front propagation from hot spots, Combust.Flame 133 (2003) 63-74.

[25] P. Dai, Z. Chen, S. Chen, Y. Ju, Numerical experiments on reaction front propagation in n-heptane/air mixture with temperature gradient, P Combust Inst 35 (2015) 3045-3052.

[26] A. Robert, S. Richard, O. Colin, T. Poinsot, LES study of deflagration to detonation mechanisms in a downsized spark ignition engine, Combust.Flame 162 (2015) 2788-2807.

[27] H. Terashima, M. Koshi, Mechanisms of strong pressure wave generation in end-gas autoignition during knocking combustion, Combust.Flame 162 (2015) 1944-1956.

[28] N. Kawahara, E. Tomita, Y. Sakata, Auto-ignited kernels during knocking combustion in a spark-ignition engine, P Combust Inst 31 (2007) 2999-3006.

[29] Y. Qi, Z. Wang, J. Wang, X. He, Effects of thermodynamic conditions on the end gas combustion mode associated with engine knock, Combust.Flame 162 (2015) 4119-4128.

[30] H. Wei, J. Zhao, L. Zhou, D. Gao, Z. Xu, Effects of the equivalence ratio on turbulent flame-shock interactions in a confined space, Combust.Flame 186 (2017) 247-262.

[31] H. Wei, J. Zhao, X. Zhang, J. Pan, J. Hua, L. Zhou, Turbulent flame-shock interaction inducing end-gas autoignition in a confined space, Combust.Flame 204 (2019) 137-141.

[32] V.N. Gamezo, T. Ogawa, E.S. Oran, Flame acceleration and DDT in channels with obstacles: Effect of obstacle spacing, Combust.Flame 155 (2008) 302-315.

[33] B. Varatharajan, F.A. Williams, Chemical-kinetic descriptions of high-temperature ignition and detonation of acetylene-oxygen-diluent systems, Combust.Flame 124 (2001) 624-645.

[34] H. Peng, Y. Huang, R. Deiterding, Z. Luan, F. Xing, Y. You, Effects of jet in crossflow on flame acceleration and deflagration to detonation transition in methane-oxygen mixture, Combust.Flame 198 (2018) 69-80.

[35] G.B. Goodwin, R.W. Houim, E.S. Oran, Effect of decreasing blockage ratio on DDT in small channels with obstacles, Combust.Flame 173 (2016) 16-26.

[36] L. Kagan, M. Liberman, G. Sivashinsky, Detonation initiation by a hot corrugated wall, P Combust Inst 31 (2007) 2415-2420.

[37] D. Bradley, M. Lawes, K. Liu, Turbulent flame speeds in ducts and the deflagration/detonation transition, Combust.Flame 154 (2008) 96-108.

[38] J. Chao, J.H.S. Lee, The propagation mechanism of high speed turbulent deflagrations, ShWav 12 (2003) $277-289$.

[39] M. Kuznetsov, V. Alekseev, Y. Yankin, S. Dorofeev, Slow and fast deflagrations in hydrocarbon-air mixtures, Combust Sci Technol 174 (2002) 157-172.

[40] C.J. Sun, C.J. Sung, L. He, C.K. Law, Dynamics of weakly stretched flames: Quantitative description and extraction of global flame parameters, Combust.Flame 118 (1999) 108-128.

[41] V. Bychkov, V.y. Akkerman, G. Fru, A. Petchenko, L.-E. Eriksson, Flame acceleration in the early stages of burning in tubes, Combust.Flame 150 (2007) 263-276.

[42] D.M. Valiev, V.y. Akkerman, M. Kuznetsov, L.-E. Eriksson, C.K. Law, V. Bychkov, Influence of gas compression on flame acceleration in the early stage of burning in tubes, Combust.Flame 160 (2013) 97-111.

[43] G. Ciccarelli, C.T. Johansen, M. Parravani, The role of shock-flame interactions on flame acceleration in an obstacle laden channel, Combust.Flame 157 (2010) 2125-2136.

[44] A. Petchenko, V. Bychkov, V.y. Akkerman, L.-E. Eriksson, Flame-sound interaction in tubes with nonslip walls, Combust.Flame 149 (2007) 418-434.

[45] Y. Wu, Q. Zheng, C. Weng, An experimental study on the detonation transmission behaviours in acetylene-oxygenargon mixtures, Energy 143 (2018) 554-561. 
[46] L. Wang, H. Ma, Z. Shen, Z. Fan, Detonation characteristics of stoichiometric H-2-O-2 diluted with Ar/N-2 in smooth and porous tubes, Exp Therm Fluid Sci 91 (2018) 345-353.

[47] Y. Fortin, J. Liu, J.H.S. Lee, Mach reflection of cellular detonations, Combust.Flame 162 (2015) 819-824.

[48] R. Mevel, D. Davidenko, F. Lafosse, N. Chaumeix, G. Dupre, C.-E. Paillard, J.E. Shepherd, Detonation in hydrogennitrous oxide-diluent mixtures: An experimental and numerical study, Combust.Flame 162 (2015) 1638-1649.

[49] S. Gallier, F. Le Palud, F. Pintgen, R. Mevel, J.E. Shepherd, Detonation wave diffraction in H-2-O-2-Ar mixtures, P Combust Inst 36 (2017) 2781-2789.

[50] B. Zhang, N. Mehrjoo, H.D. Ng, J.H.S. Lee, C. Bai, On the dynamic detonation parameters in acetylene-oxygen mixtures with varying amount of argon dilution, Combust.Flame 161 (2014) 1390-1397. 